\title{
Effect of Prestress on the Stability of Electrode-Electrolyte Interfaces during Charging in Lithium Batteries
}

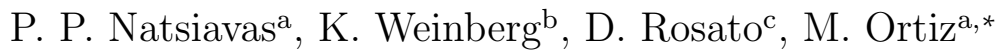 \\ ${ }^{a}$ Division of Engineering and Applied Science, California Institute of Technology \\ Pasadena, CA91125, USA \\ ${ }^{b}$ Lehrstuhl für Festörpermechanik, Universität Siegen, D-57068 Siegen, Germany \\ ${ }^{c}$ VHIT S.p.A. - Società Unipersonale Bosch Group Italy (EAD2), Strada Vicinale delle \\ Sabbione, 5, 26010 Offanengo (CR), Italia
}

\begin{abstract}
We formulate a model of the growth of electrode-electrolyte interfaces in lithium batteries in the presence of an elastic prestress. The model accounts for the kinetics of $\mathrm{Li}^{+}$transport through a solid electrolyte and, within the interface, for the kinetics of $\mathrm{Li}^{+}$adsorption by the anode, electrostatics, and the elastic field. We specifically account for the effect of the elastic field through an asymptotic analysis of a nearly flat interface between two semi-infinite elastic bodies. We use the model as a basis for assessing the effect of prestress on the stability of planar growth and the potential of prestress as a means of suppressing the formation of deleterious dendrites. We present a linear stability analysis that results in explicit analytical expressions for the dependence of growth rates, and of the critical unstable wavelength for the interfacial roughening, on the state of prestress and on fundamental parameters such as surface diffusivities, surface energy, deposition kinetics, and elastic moduli. Finally, we examine the model in the light of experimental observations concerned with the effect of applied pressure on a lithium/dioxolane-dimethoxy ethane electrolyte systems. With reasonable choices of parameters and some calibration, the model accounts for the observation that a modest applied pressure indeed results in a substantial reduction in the roughening of the lithium surface during cycling.
\end{abstract}

\footnotetext{
${ }^{*}$ Corresponding author

E-mail address: ortiz@aero.caltech.edu (M. Ortiz).
}

Preprint submitted to JMPS

April 7, 2016

(C) 2016. This manuscript version is made available under the Elsevier user license http://www.elsevier.com/open-access/userlicense/1.0/ 


\section{Introduction}

The present work is concerned with the assessment of the effect of prestress on the stability of electrode-electrolyte interfaces in lithium batteries and, in particular, of the potential of prestress as a means of suppressing the formation of deleterious dendrites. Lithium compounds are widely used as a electrode materials for rechargeable batteries due to the high electropositivity and low weight of metallic lithium. During battery operation, lithium at the anode, the negative electrode, gives up electrons to become $\mathrm{Li}^{+}$, which dissolves into the electrolyte. The electrons move through the electrical circuit to the positive electrode, the cathode, as shown in Fig. 1. In a recharging battery this process is reversed and lithium receives current from an external generator. In order to avoid confusion, we will refer to the electrode on the left of Fig. 1 as the negative electrode and to the one on the right as the positive electrode, since this characterization is correct during both charging and discharging cycles.

Lithium cations, during recharge, move from the positive electrode to the negative one. If the lithium deposition is not uniform across the electrode surface, it can form protrusions, and even dendrites, that eventually may short the battery $[7,10,30]$. Because reactions between the lithium metal and the electrolytes are exothermic, large surface areas also pose risk of overheating, or thermal runaway [69, 67]. Dendrites at the lithium electrode have been reported to grow from one electrode to the other through the electrolyte $[1,42,71,15,2]$. One of the mechanisms that trigger and mediate dendrite formation is thought to be the localized current surge at dendrite tips [12]. The size of dendrites ranges between 2-2,000 $\mu \mathrm{m}$ depending on the material system, cf. Figs. 2 and 3.

Dendritic lithium deposits on the battery electrode after being subjected to multiple charges and recharges were first observed by Epelboin et al. [22] and later on by Yoshimatsu et al. [74] and others. Dolle et al. [17] reported dendritic growth of the interface on lithium polymer batteries and confirmed direct correlation between current density and dendrite formation. Other authors $[55,11,64,34,72,73]$ studied the deposition mechanisms of metals in non-aqueous battery systems. Barton and Bockris [5] and Diggle et al. [16] put forth the first comprehensive model of dendrite growth. Improvement in lithium cycling efficiency using additives is discussed in other works [61, 24, 


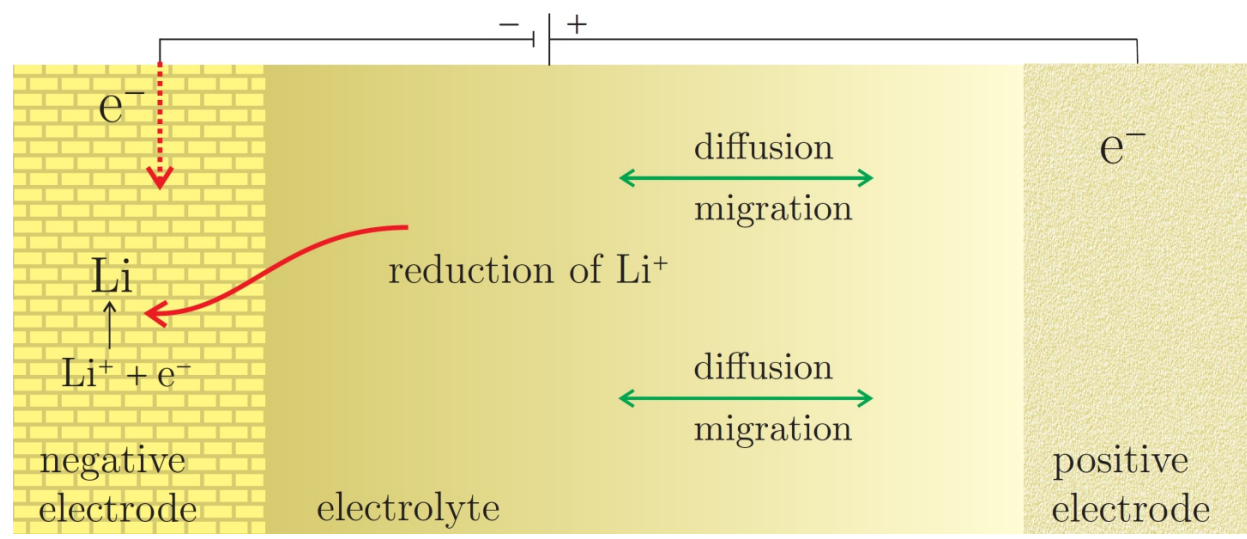

Figure 1: Charging process at the lithium anode.

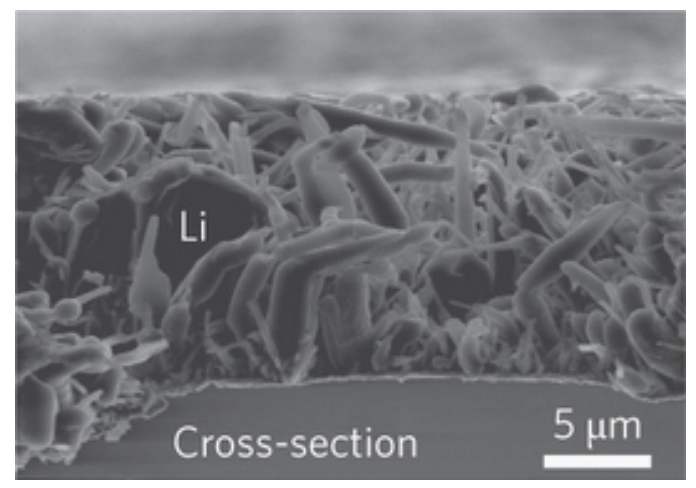

Figure 2: SEM characterization of lithium deposition on a copper substrate: at the electrode ramified growth of mossy lithium/dendrites is clearly observed. Photograph from [77]. 




Figure 3: Dendrites in real battery cell. From [3].

$60]$.

Studies with a more mechanical focus include those of Ely and García [20], Nishida et al. [49], and Monroe and Newman [42]. The latter study conclude that interfacial roughening is mechanically suppressed when the separator shear modulus is about twice that of lithium. Mikhaylik et al. [40] experimentally confirmed that application of pressure between the electrodes lowers the roughness of the lithium anode, an effect first posited by Hirai et al. [33]. However, a fundamental understanding of the effect of pressure and, more generally, prestress on the stability of lithium-electrolyte interfaces appears to be lacking at present, which hampers the practical exploitation of prestress in battery design.

The objective of the present study is to ascertain conditions under which an electrode-electrolyte interface grows in a planar geometry and how such conditions are influenced by the elastic field of built-in prestress. Of particular interest is to elucidate the manner in which elastic prestress can be utilized in the design of lithium batteries in order to eliminate dendrite formation and its deleterious effects during the lifetime of the cell. We base our analysis on a model of interfacial growth that accounts for the kinetics of $\mathrm{Li}^{+}$transport through the solid electrolyte (e. g., LIPON) and within the interface, the kinetics of $\mathrm{Li}^{+}$adsorption by the anode, electrostatics, and the elastic field. These fields are coupled due to pressure-assisted diffusion, swelling of the intercalated electrolyte, and the Maxwell stress of the elec- 
trostatic field, and contribute jointly to the chemical potential driving the motion of interface. In particular, the elastic field is sensitive to the shape of the interface, with the result that its roughening may be self-sustaining and eventually lead to the runaway formation of dendrites.

In this work, we account for this feedback effect through an asymptotic analysis of the elastic field of a nearly flat interface between two semi-infinite elastic bodies. Similar asymptotic analyses of nearly flat surfaces and interfaces between elastic bodies have been carried out by Srolovitz [63] and by Gao [27, 28]. Linear stability analysis then results in explicit analytical expressions for the dependence of growth rates, and of the critical unstable wavelength for the interfacial roughening, on the state of stress and on fundamental parameters such as surface diffusivities, surface energy, deposition kinetics, and elastic moduli.

The paper is organized as follows. We begin by formulating the mathematical model and deriving the governing equations of lithium transport through the solid electrolyte from its bulk free-energy density. The equation that describes the evolution of the surface is also derived using interfacial energy and kinetics. Specifically, the excess elastic energy due to the coupling between interfacial waviness and a built-in state of prestress is obtained analytically in the limit of nearly-flat interfaces. The requisite interfacial Green's function is derived using Fourier transform methods and subsequently verified against analytical expressions from Pan and Yuan [54] and finite-element simulations using commercial software. We begin by demonstrating the possibility of stable and unstable growth by solving directly the evolution equation for the interfacial profile using finite-differences and an implicit time-stepping scheme. A linear stability analysis is then performed in order to characterize the stability regimes and the conditions that influence them, including the effect of prestress. The extent of prestress stabilization in the specific LiLIPON material system is assessed through a parametric study. Finally, we examine the model in the light of the experimental observations of Mikhaylik et al. [40] concerned with the effect of pressure on a lithium/dioxolanedimethoxy ethane electrolyte system.

\section{Formulation of the model}

We seek to characterize the evolution of an anode-electrolyte interface profile

$$
x_{3}=h\left(x_{1}, x_{2}, t\right)
$$


during the early stages of charge. In this representation, we assume the interface to be shallow, thus representable as a graph, and we describe its profile by means of a height function $h$. In so doing, we choose Cartesian axes such that $\left(x_{1}, x_{2}\right)$ span the interface and $x_{3}$ is transverse to it. The physical processes which are assumed to contribute to the growth are i) surface diffusion, capillarity-driven by particle exchange between the anode and the electrolyte; ii) electrochemical deposition; and iii) energetic forces associated with the elastic and electrostatic fields of the anode/substrate.

In this section, we proceed to formulate models for each of these processes in turn. We note that the formulation of high-fidelity models for solid electrolytes, the electrode-electrolyte interface, and their evolution is an active area of research that is beyond the scope of this paper. Conveniently, for purposes of linearized stability analysis in the asymptotic regime of nearlyflat interfaces of interest here, the governing equations are greatly simplified and reduced and the details of the models wash away, making the analysis relatively 'model-insensitive'. However, for completeness and to provide context, we begin by sketching typical forms of the equations that govern the elastic, electrostatic and concentration fields in a solid electrolyte.

\subsection{Bulk free-energy and lithium transport through electrolyte}

For definiteness, we assume the model electrolytes to be solid-state. We consider the following bulk free energy per unit volume in the electrolyte:

$$
\begin{aligned}
A(\boldsymbol{\epsilon}, T, c, \varphi) & =\frac{1}{2} c_{i j k l}\left(\epsilon_{i j}-\epsilon_{i j}^{*}-\frac{\Omega c}{3} \delta_{i j}\right)\left(\epsilon_{k l}-\epsilon_{k l}^{*}-\frac{\Omega c}{3} \delta_{k l}\right) \\
& +\frac{\varepsilon}{2}|\nabla \varphi|^{2}+\varepsilon\left(\varphi,{ }_{i} \varphi,{ }_{j}-\frac{|\nabla \varphi|^{2}}{2} \delta_{i j}\right) \epsilon_{i j} \\
& +\mu_{0} c+R T\left(c \log \left(\frac{c}{c_{0}}\right)+\left(c_{0}-c\right) \log \left(1-\frac{c}{c_{0}}\right)\right) .
\end{aligned}
$$

Here, $c_{i j k l}$ are the elastic moduli, $\epsilon_{i j}^{*}$ is prescribed prestrain, $\Omega$ is the partial molar volume of ion dissolved in the solid electrolyte $\left[\mathrm{m}^{3} / \mathrm{mol}\right] ; c$ is the lithium concentration, $c_{0}$ is a reference concentration. The electric permittivity is $\varepsilon[\mathrm{Farad} / \mathrm{m}] ; \varphi$ is the local electrostatic potential [V]. The ideal chemical potential per mole is $\mu_{0}[\mathrm{~J} / \mathrm{mol}] ; R$ is the ideal gas constant, $T$ the absolute temperature. Additional free energy contributions need to be accounted for in systems in which phase segregation occurs (cf., e. g., [47, 26, 6]).

The first term in the bulk free-energy density (2) accounts for the elastic field, including a prestrain and the effect of swelling due to the intercalating 
lithium ions. The second line of (2) summarizes the electric field contributions, i. e., the permittivity and electro-mechanical interaction through the Maxwell stress. Thus, the mechanical stress follows as

$$
\sigma_{i j}=\frac{\partial A}{\partial \epsilon_{i j}}=c_{i j k l}\left(\epsilon_{k l}-\epsilon_{k l}^{*}-\frac{\Omega c}{3} \delta_{k l}\right)+\varepsilon\left(\varphi, i \varphi, j-\frac{|\nabla \varphi|^{2}}{2} \delta_{i j}\right) .
$$

We note that, for the systems of interest, the bulk free-energy can be conveniently simplified, without significant loss of accuracy, by neglecting the terms with the electric permittivity $\varepsilon$. Thus, for the example of LIPON with $\varepsilon \sim 10^{-7} \mathrm{~F} / \mathrm{m}[38]$ and with $1 \%$ of straining at $1000 \mathrm{~V}$ the elastic stress is estimated to be in the order of 100 times the Maxwell stress.

The last line in (2) accounts for the entropy of mixing of the intercalated lithium ions. The chemical potential of the lithium ion concentration follows as

$$
\mu=\frac{\partial A}{\partial c}=\mu_{0}+R T\left(\log \left(\frac{c}{c_{0}}\right)-\log \left(1-\frac{c}{c_{0}}\right)\right)-\Omega p,
$$

where $p=\frac{1}{3} \sigma_{k k}$ is the mean stress. We note that the chemical potential (4) accounts for the coupling with the elastic field through the pressure.

General equations governing the lithium transport in the electrolyte region may be formulated as follows (cf., e. g., [8, 18, 37, 56] for alternative formulations). Conservation of mass for the concentration $c$ demands that

$$
\frac{\partial c}{\partial t}+\nabla \cdot \boldsymbol{J}=0
$$

where $\boldsymbol{J}$ is the vector flux of $\mathrm{Li}^{+}$ions through the electrolyte in moles per unit time per unit area. Charge conservation in turn requires

$$
\frac{\partial \varrho_{e}}{\partial t}+\nabla \cdot \boldsymbol{i}=0
$$

with $\boldsymbol{i}$ being the total current density $\left[\mathrm{A} / \mathrm{m}^{2}\right]$. The charge density $\varrho_{e}$ always sums up to zero due to charge neutrality. Mechanical equilibrium finally requires that

$$
\nabla \cdot \boldsymbol{\sigma}=\mathbf{0}
$$

The preceding conservation equations are closed by putting forth appropriate materials laws. Thus, the ion flux is composed of diffusive and electric contributions,

$$
\boldsymbol{J}=-M c \nabla \mu+\frac{t_{+}}{z_{+} F} \boldsymbol{i}
$$


where $z_{ \pm}$is the charge number per ion (for lithium $z_{+}=1$ ), $F$ is Faraday's constant and $t_{ \pm}$are ionic transference numbers indicating the fraction of the total current carried by the ions or electrons $\left(t_{+}+t_{-}=1\right)$. The mobility of lithium depends on concentration as

$$
M(c)=\frac{D_{s}}{R T}\left(1-\frac{c}{c_{\max }}\right),
$$

where $D_{s}$ is the corresponding chemical diffusion coefficient. In the case of $c=c_{\max }$, all possible sites are occupied by lithium ions and the mobility coefficient reduces to zero. With conductance $\kappa$ the electric current follows as

$$
\boldsymbol{i}=-\kappa \nabla \varphi+\frac{\kappa t_{-}}{z_{+} F} \nabla \mu
$$

Finally, the mechanical stress may be assumed to obey Hooke's law of anisotropic linear elasticity.

For a solid state electrolyte, the concentration gradients in the bulk of the material are negligible since the concentration of the $\mathrm{Li}^{+}$remains ostensibly constant through the electrolyte (cf., e. g., [48] for a discussion). In this case, ion flux and electric current are proportional to $\nabla \varphi$ and the balance equations (5) and (6) reduce trivially to the Laplace equation

$$
\Delta \varphi=0
$$

Assuming Dirichlet boundary conditions at the electrode-electrolyte interfaces, we obtain from equation (11) that the potential is a linear function of position within the electrolyte, corresponding to a constant electric field. We note, however, that at the lithium electrode interface the potential may drop and even show interfacial impedances [39].

\subsection{Electrode-electrolyte interface energy and kinetics}

Next, we consider the kinetics of a nearly flat electrode-solid electrolyte interface resulting from interfacial mass transport. We suppose that the kinetics of the interface is governed by three main mechanisms: i) interfacial diffusion; ii) electrochemical deposition; and iii) the chemical potential resulting from interfacial and bulk free-energies, including the coupling to the elastic field. We proceed to consider these contributions in turn. We specifically follow the extensive body of work on continuum and atomistic models of kinetic surface and interfacial growth with surface relaxation $[35,13,29,57,58,59,62,76,52]$. 
Mass conservation demands that

$$
\frac{\partial h}{\partial t}=\Omega j_{m}-\nabla \cdot \boldsymbol{j}_{p},
$$

where $j_{m}\left[\mathrm{~mol} /\left(\mathrm{m}^{2} \mathrm{~s}\right)\right]$ is the mass flux of $\mathrm{Li}^{+}$from the electrolyte to the electrode and $\boldsymbol{j}\left[\mathrm{m}^{2} / \mathrm{s}\right]$ is the interfacial mass flux. We assume the latter to be of the form

$$
\boldsymbol{j}_{p}=-\nabla\left(D_{1} h+D_{2} \nabla^{2} h\right),
$$

where $\nabla$ denotes the in-plane gradient and $D_{1}\left[\mathrm{~m}^{2} / \mathrm{s}\right]$ and $D_{2}\left[\mathrm{~m}^{4} / \mathrm{s}\right]$ are surface diffusivities. The first term in (13) accounts for Mullins diffusion by particle exchange between the electrode and the electrolyte, whereas the second term models Herring-Mullins capillarity-driven interfacial diffusion, cf. [43, 36, 32].

We additionally assume the mass flux of $\mathrm{Li}^{+}$from the electrolyte to the electrode to be governed by a linear kinetic equation of the form

$$
j_{m}=\frac{K d}{R T \Omega} z_{+} F \eta_{s},
$$

where $\eta_{s}$ denotes the overpotential along the electrode's surface, $d$ is the atomic interplanar distance in the solid electrode, and $K$ is an equilibrium exchange rate coefficient for mass transfer between the electrolyte and the electrode. Eq. (14) is a linearization of the Butler-Volmer equation, which is frequently used in electrochemistry to describe the transport of ions across an interface (cf., e. g., $[37,42,48]$ ). We recall that the Butler-Volmer equation gives the electric current density as

$$
i_{\text {ion }}=i_{0}\left(\exp \left(\frac{\alpha_{a} F}{R T} \eta_{s}\right)-\exp \left(-\frac{\alpha_{c} F}{R T} \eta_{s}\right)\right),
$$

where $i_{0}$ is the $\mathrm{Li}^{+}$concentration dependent exchange current density $\left[\mathrm{A} / \mathrm{m}^{2}\right]$, which is an interfacial property and should not be confused with the charging current density, and $\alpha_{a}$ and $\alpha_{c}$ are the anodic and cathodic transfer coefficients, respectively, with $\alpha_{a}+\alpha_{c}=1$. The surface overpotential $\eta_{s}$ is the difference

$$
\eta_{s}=\varphi^{\mathrm{S}}-\varphi^{E}-\frac{\mu^{\mathrm{E}}-\mu^{\mathrm{S}}}{z_{+} F},
$$

where the label $E$ refers to the electrolyte and the label $S$ refers to the solid electrode. For small variations in $i_{\text {ion }}$ about a reference state, as envisioned here, the Butler-Volmer equation can be linearized, leading to (14). 
Lastly, we account for mass deposition driven by the configurational forces attendant to the coupling of the elastic field of the electrode-electrolyte system to the shape of the interface. In order to identify the corresponding driving force, we begin by noting that the elastic energy $E[h]$ of the system per unit area of the interface at equilibrium depends, configurationally, on the interface profile $h\left(x_{1}, x_{2}, t\right)$. The corresponding chemical potential is given by the functional derivative

$$
\mu_{h}=\Omega \frac{\delta E}{\delta h} .
$$

Assuming, in addition, linear kinetics, the corresponding mass deposition rate $j_{h}$ follows as

$$
j_{h}=-\frac{K d}{R T \Omega} \mu_{h}
$$

Taking into account all the mechanisms of mass transport just described, equation (12) becomes

$$
\frac{\partial h}{\partial t}=\nabla^{2}\left(D_{1} h+D_{2} \nabla^{2} h\right)+\frac{K d}{R T} z_{+} F \eta_{s}-\frac{K d}{R T} \frac{\delta E}{\delta h} .
$$

This equation describes the evolution in time of the surface profile. In order to render (19) completely explicit, an expression for the dependence of $E$ on $h$ is required. This dependence is derived next by recourse to an asymptotic analysis in the limit of nearly-flat interfaces, cf. [63, 27, 28].

\subsection{The nearly-flat electrode-electrolyte interface}

We wish to estimate the dependence of the elastic energy of an idealized electrode-electrolyte system on the - possibly slightly non-planarprofile $h\left(x_{1}, x_{2}, t\right)$ of the interface. We derive this estimate asymptotically in the limit of a shallow, or nearly-flat interface. Conveniently, this asymptotic limit suffices to establish the linear stability properties of a planar interface, which is the main focus of the present work.

As already mentioned, the focus on linearized stability and nearly-flat interfaces has the effect of greatly simplifying the governing equations exemplified in Section 2, rendering the analysis relatively model-free. Thus, the governing equations enter the stability analysis linearized, which streamlines the equations greatly. Perhaps more importantly, owing to the scalar nature of the electrostatic potential and of the concentration field, their variation upon the introduction of a slight waviness in the interface is asymptotically of 
second-order and can be neglected to a first approximation. This additional reduction confers the analysis great simplicity and generality, as, to leading order, the effect of the interface waviness is strictly an elastic effect. In consequence, the details of the electrostatic and diffusion models presented in Section 2, and of alternative models thereof, turn out to be irrelevant for present purposes. Here again, this simplification has the beneficial and appealing effect of rendering the analysis general and relatively model-free.

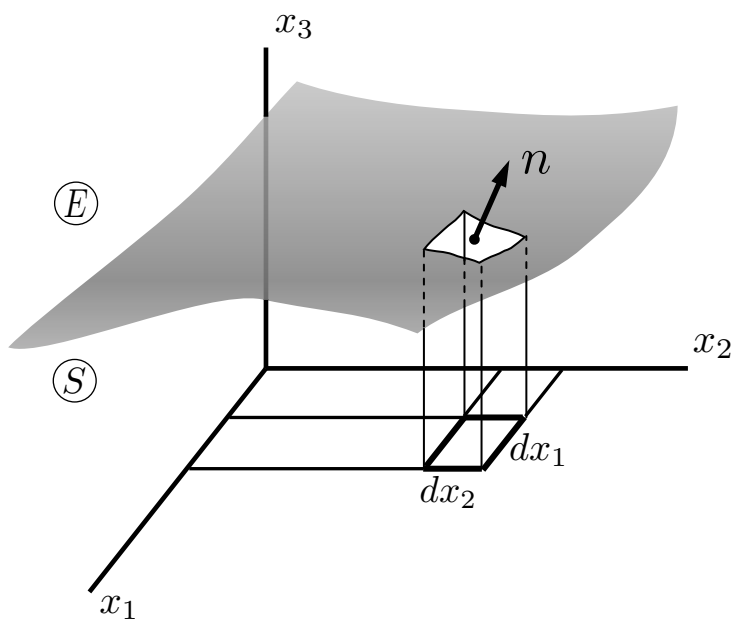

Figure 4: Electrolyte-electrode domain and area projection on the $x_{1}-x_{2}$ plane.

Since we are only interested in the local behavior of the interface, we may idealize the domain of the electrolyte as being $h\left(x_{1}, x_{2}, t\right)<x_{3}<+\infty$, and the domain of the lithium electrode as being $-\infty<x_{3}<h\left(x_{1}, x_{2}, t\right)$, see Fig. 4 . To leading order in $|\nabla h|$, the corresponding unit normal to the interface is given by

$$
\boldsymbol{n} \sim(-h, 1,-h, 2,1),
$$

and the element of area by

$$
d A \sim\left(1+\frac{1}{2}|\nabla h|^{2}\right) \mathrm{d} x_{1} \mathrm{~d} x_{2} .
$$

From this identity, the interfacial energy difference relative to the planar interface follows directly to leading order as

$$
E^{\text {int }}[h]=\int \frac{\gamma}{2}|\nabla h|^{2} \mathrm{~d} x_{1} \mathrm{~d} x_{2},
$$


where $\gamma$ is the interfacial energy per unit area.

In addition to the concentration and electrostatic fields, described in section 2.1, a prestressed planar interface carries an elastic field. We specifically assume piecewise uniform and equilibrated prestrain field $\epsilon_{i j}^{*}(\boldsymbol{x}, t)$ and residual stress, or prestress, field $\sigma_{i j}^{*}(\boldsymbol{x}, t)$ at zero $\mathrm{Li}^{+}$concentration. From compatibility, equilibrium and Hooke's law, we specifically have

$$
\begin{array}{ll}
\epsilon_{i j}^{*}=\epsilon_{i j}^{* E}, & x_{3}>0 \\
\epsilon_{i j}^{*}=\epsilon_{i j}^{* S}, & x_{3}<0 \\
\sigma_{i j}^{*}=-c_{i j k l}^{E} \epsilon_{k l}^{* E} \equiv \sigma_{i j}^{* E}, & x_{3}>0 \\
\sigma_{i j}^{*}=-c_{i j k l}^{*} \epsilon_{k l}^{* S} \equiv \sigma_{i j}^{* S}, & x_{3}<0 \\
\sigma_{i 3}^{* E}-\sigma_{i 3}^{* S}=0, & x_{3}=0 .
\end{array}
$$

Thus, for the planar interface the elastic field consists of equilibrated constant residual stresses $\sigma_{i j}^{* E}$ and $\sigma_{i j}^{* S}$ in the electrolyte and the solid electrode, respectively.

Deviations from the planar profile modify the planar elastic field (23) to leading order in $|\nabla h|$. The corresponding correction displacement field $\boldsymbol{u}$ satisfies the problem

$$
\begin{array}{lr}
\sigma_{i j, j}^{S}(u)=0, & x_{3}<h\left(x_{1}, x_{2}, t\right), \\
\sigma_{i j, j}^{E}(u)=0, & x_{3}>h\left(x_{1}, x_{2}, t\right), \\
\sigma_{i 3}^{E}(u)-\left(\sigma_{i \beta}^{* E}+\sigma_{i \beta}^{E}(u)\right) h,_{\beta}= & \\
\sigma_{i 3}^{S}(u)-\left(\sigma_{i \beta}^{* S}+\sigma_{i \beta}^{S}(u)\right) h,_{\beta}, & x_{3}=h\left(x_{1}, x_{2}, t\right), \\
u_{i} \rightarrow 0, & x_{3} \rightarrow \pm \infty,
\end{array}
$$

where, here and subsequently, Greek indices take values in $\{1,2\}$, and we write

$$
\begin{aligned}
& \sigma_{i j}^{S}(u)=c_{i j k l}^{S} \epsilon_{k l}^{S}(u) \\
& \sigma_{i j}^{E}(u)=c_{i j k l}^{E} \epsilon_{k l}^{E}(u) .
\end{aligned}
$$

Introducing the representation

$$
u_{i}\left(x_{1}, x_{2}, x_{3}, t\right)=v_{i}\left(x_{1}, x_{2}, x_{3}-h\left(x_{1}, x_{2}, t\right), t\right),
$$

we obtain

$$
\begin{aligned}
& u_{i, \alpha}=v_{i, \alpha}-v_{i, 3} h,_{\alpha}, \\
& u_{i, 3}=v_{i, 3},
\end{aligned}
$$


or

$$
u_{i, j}=v_{i, j}-v_{i, 3} h,{ }_{j} \delta_{j \beta}
$$

whence

$$
\epsilon_{i j}(u)=\epsilon_{i j}(v)-\frac{1}{2}\left(v_{i, 3} \delta_{\beta j}+v_{j, 3} \delta_{\beta i}\right) h, \beta
$$

and

$$
\sigma_{i j}(u)=\sigma_{i j}(v)-c_{i j k \delta} v_{k, 3} h,_{\delta} .
$$

In this representation, the equilibrium problem becomes, to first order,

$$
\begin{array}{lr}
\sigma_{i j, j}^{S}(v)=0, & x_{3}<0, \\
\sigma_{i j, j}^{E}(v)=0, & x_{3}>0, \\
\sigma_{i 3}^{E}(v)-\sigma_{i \beta}^{* E} h,_{\beta}=\sigma_{i 3}^{S}(v)-\sigma_{i \beta}^{* S} h, \beta, & x_{3}=0, \\
v_{i} \rightarrow 0, & x_{3} \rightarrow \pm \infty .
\end{array}
$$

The attendant change in elastic energy (cf. Appendix A) results as

$$
E^{\mathrm{ela}}[h]=\int_{\mathbb{R}^{2}} \int_{\mathbb{R}^{2}} \frac{1}{2} G_{i k}\left(\boldsymbol{x}-\boldsymbol{x}^{\prime}\right) \llbracket \sigma_{i \beta}^{*} \rrbracket \llbracket \sigma_{k \delta}^{*} \rrbracket h, \beta_{\beta}(\boldsymbol{x}) h,_{\delta}\left(\boldsymbol{x}^{\prime}\right) d \boldsymbol{x} d \boldsymbol{x}^{\prime},
$$

where

$$
\llbracket \sigma_{i j}^{*} \rrbracket=\sigma_{i j}^{* E}-\sigma_{i j}^{* S},
$$

is the jump in the residual stress tensor at the interface, and $\boldsymbol{G}\left(\boldsymbol{x}, \boldsymbol{x}^{\prime}\right)$ is the interfacial Green's function, derived explicitly in Appendix B. From these identities, the total elastic energy difference relative to the planar interface finally follows as

$$
\begin{aligned}
E[h] & =E^{\mathrm{int}}[h]+E^{\mathrm{ela}}[h]=\int_{\mathbb{R}^{2}} \frac{\gamma}{2}|\nabla h|^{2} d \boldsymbol{x} \\
& +\int_{\mathbb{R}^{2}} \int_{\mathbb{R}^{2}} \frac{1}{2} G_{i k}\left(\boldsymbol{x}-\boldsymbol{x}^{\prime}\right) \llbracket \sigma_{i \beta}^{*} \rrbracket \llbracket \sigma_{k \delta}^{*} \rrbracket h, \beta_{\beta}(\boldsymbol{x}) h, \delta\left(\boldsymbol{x}^{\prime}\right) d \boldsymbol{x} d \boldsymbol{x}^{\prime} .
\end{aligned}
$$

Note here that the total energy difference vanishes for the case of a planar interface, i. e., $h,_{\alpha}=0$, as required. The requisite functional derivative in the equation of evolution (19) may now be written explicitly as

$$
\frac{\delta E}{\delta h}(\boldsymbol{x})=-\gamma \nabla^{2} h(\boldsymbol{x})-\int_{\mathbb{R}^{2}} G_{i k, \beta}\left(\boldsymbol{x}-\boldsymbol{x}^{\prime}\right) \llbracket \sigma_{i \beta}^{*} \rrbracket \llbracket \sigma_{k \delta}^{*} \rrbracket h, \delta_{\delta}\left(\boldsymbol{x}^{\prime}\right) d \boldsymbol{x}^{\prime},
$$

which renders (19) completely explicit. 
It bears emphasis that the elastic field attendant to the introduction of a slight waviness in the interface is induced, to first order, by the interfacial tractions $\llbracket \sigma_{i \beta}^{*} \rrbracket h,_{\beta}$. These tractions arise as a direct result of the vector character of the elastic field. By contrast, the electrostatic and concentration fields are scalar and, therefore, a similar treatment shows that the corresponding interfacial sources induced by a slight waviness in the interface vanish to first order. Consequently, as already mentioned, the perturbation of the electrostatic and concentration fields is asymptotically of second-order and can be neglected to a first approximation.

\subsection{Initial boundary value problem and model calibration}

The equation that governs the evolution of the interfacial height profile $h\left(x_{1}, x_{2}, t\right)$ is finally obtained by inserting the configurational driving force (35) into (19). We note that the resulting evolution equation is linear and, hence, the principle of superposition applies. In particular, the second term on the right-hand side of (19) induces a uniform translational velocity

$$
V=\frac{K d}{R T} z_{+} F \eta_{s}
$$

Effecting the change of variables $h \rightarrow h-V t$, the term drops out. For ease of handling, we additionally reparameterize the resulting evolution of equation in the form

$$
\frac{\partial h}{\partial t}=\left(D_{1}+D_{3}\right) \nabla^{2} h+D_{2} \nabla^{4} h+D_{4} \int_{\mathbb{R}^{2}} G_{i k, \beta}\left(\boldsymbol{x}-\boldsymbol{x}^{\prime}\right) \llbracket \sigma_{i \beta}^{*} \rrbracket \llbracket \sigma_{k \delta}^{*} \rrbracket h, \delta\left(\boldsymbol{x}^{\prime}\right) d \boldsymbol{x}^{\prime},
$$

where

$$
D_{3}=\frac{\Omega K d \gamma}{R T}, \quad D_{4}=\frac{\Omega K d}{R T}=\frac{D_{3}}{\gamma},
$$

and the interface Green's function is given explicitly in Fourier form in Appendix A. The initial-value problem is fully defined by specifying the domain of analysis, boundary conditions and the initial profile $h\left(x_{1}, x_{2}, 0\right)$.

In order to calibrate the model for specific materials systems, we need to estimate the diffusion coefficients $D_{1}$ and $D_{2}$. Following Mullins [43], we may expect the diffusion constant for particle exchange between the electrode and the electrolyte to be of the form

$$
D_{1}=\frac{p_{0} \gamma \Omega_{m}^{2}}{(2 \pi M)^{1 / 2}\left(k_{B} T\right)^{3 / 2}},
$$


where is an equilibrium vapor pressure, $\gamma$ the surface energy per unit area on the lithium-electrolyte interface, $\Omega_{m}$ the molecular volume, $M$ the weight of a molecule, $k_{B}=1.3806 \cdot 10^{-23} \mathrm{~J} / \mathrm{K}$ the Boltzmann constant, and $T$ is the absolute temperature. Likewise, Herring-Mullins [32] capillarity-driven interfacial diffusion constant accounts for a surface diffusion with coefficient $D_{s}$ and results in

$$
D_{2}=\frac{D_{s} \gamma \Omega_{m}^{2} \nu}{k_{B} T}
$$

where $\nu$ the number of atoms per unit area. It should be noted that $D_{2}$ can be positive or negative.

\subsection{Example: Li-LIPON system}

We proceed to demonstrate the possibility of stable and unstable growth in a Li-LIPON system by solving directly the governing evolution equation (19) for the interfacial profile using finite-differences and an implicit timestepping scheme. We specifically consider the initial-value problem defined by (37) in a square domain with periodic boundary conditions and initial profile

$$
h\left(x_{1}, x_{2}, 0\right)=A\left(\cos \left(k_{1} x_{1}\right)+\cos \left(k_{2} x_{2}\right)\right),
$$

where $\left(k_{1}, k_{2}\right)$ are wavenumbers and $A$ an amplitude. We approximate the solutions of the initial-value problem by recourse to a five-point finite-difference scheme for the Laplacian on a regular square mesh. In addition, we compute the configurational term in (37) by means of the discrete Fourier transform using the Fourier representation of the Green's function given explicitly in Appendix A. Finally, the spatially discretized system is advanced in time by means of the Backward-Euler scheme with constant time step.

For the specific case of the Li-LIPON system under consideration, a compilation of data from various sources $[31,38,42,70,75,23,21]$ gives: $p_{0}=$ $2.1298 \cdot 10^{-8} \mathrm{~J} / \mathrm{m}^{3} ; \gamma=0.536 \mathrm{~J} / \mathrm{m}^{2}, \Omega_{m}=2.1584 \cdot 10^{-29} \mathrm{~m}^{3}, M=1.1526 \cdot 10^{-26}$ $\mathrm{kg}, D_{s}=10^{-8} \mathrm{~m}^{2} / \mathrm{s}, \nu=1.5503 \cdot 10^{19} \mathrm{~m}^{-2}$. We carry out all calculations at an absolute temperature $T=358.15 \mathrm{~K}$. These values inserted in (39) and (40) give $D_{1}=5.6835 \cdot 10^{-23} \mathrm{~m}^{2} / \mathrm{s}$ and $D_{2}=7.8288 \cdot 10^{-27} \mathrm{~m}^{4} / \mathrm{s}$, respectively.

In order to estimate $D_{3}$, we take $\Omega=1 / 1500 \mathrm{~m}^{3} / \mathrm{mol}$ to be the partial molar volume of ion dissolved in the solid electrolyte, $d=3.49 \cdot 10^{-10} \mathrm{~m}$ to be the atomic interplanar distance in the solid electrode and recall the value $R=8.314 \mathrm{~J} /(\mathrm{mol} \mathrm{K})$ of the ideal gas constant. We further estimate the kinetic 


\begin{tabular}{ccc}
\hline \hline & Lithium & LIPON \\
\hline & $c_{11}^{-}=13.5$ & $c_{11}^{+}=103.6$ \\
{$[\mathrm{GPa}]$} & $c_{12}^{-}=11.44$ & $c_{12}^{+}=44.42$ \\
& $c_{44}^{-}=8.78$ & $c_{44}^{+}=\frac{c_{11}^{+}-c_{12}^{+}}{2}=29.61$ \\
\hline \hline
\end{tabular}

Table 1: Elastic constants for lithium [9] and LIPON [31, 51, 50].

rate coefficient as

$$
K=\frac{i_{0} \Omega}{d F}
$$

with $i_{0}=30 \mathrm{~A} / \mathrm{m}^{2}$ being the exchange current density and Faraday's constant $F=96487$ Coulomb $/ \mathrm{mol}$. These values give $D_{3}=2.4874 \cdot 10^{-14} \mathrm{~m}^{2} / \mathrm{s}$. Finally, we have $D_{4}=D_{3} / \gamma=4.64067 \cdot 10^{-14} \mathrm{~m}^{4} /(\mathrm{Js})$. Clearly, $D_{3}$ is greatly in excess of $D_{1}$. Therefore, in calculations we neglect $D_{1}$.

The elastic moduli of lithium [9] and LIPON [31, 51, 50], required in the evaluation of the Green's function, are listed in Table 1.

In the absence of prestress and with wavenumbers $k_{1}=k_{2}=0.5 \mu \mathrm{m}^{-1}$, corresponding to a wavelength of $12.56 \mu \mathrm{m}$, we observe stable interface kinetics, Fig. 5a, i. e., the profile function $h$ evolves in a stable manner with the initial profile decaying exponentially and the interface becoming ostensibly planar at time of, e. g., $t=19,000 \mathrm{~s}$, Fig. $5 \mathrm{a}$ (right). By contrast, with wavenumbers $k_{1}=k_{2}=2 \mu \mathrm{m}^{-1}$ corresponding to a shorter wavelength of $3.14 \mu \mathrm{m}$, the surface evolution is unstable, with the initial profile blowing up exponentially, Fig. 5b. For a uniaxial stress jump of $\llbracket \sigma_{11}^{*} \rrbracket=100 \mathrm{MPa}$, solving the linear system with exactly the same initial conditions and parameters as before, we observe stable evolution for both wavelengths. The evolution for an initial wavelength of wavelength of $12.56 \mu \mathrm{m}$ is shown in Fig. 5c. We see from the figure that the interfacial profile becomes ostensibly planar at a time of $t=1,300 \mathrm{~s}$, Fig. 5c (right), as opposed to $t=19,000 \mathrm{~s}$ in the absence of prestress. Increasing the magnitude of the prestress results in a proportional increase of the rate of decay of the initial imperfection.

Thus, this first example illustrates the possibility of stable and unstable behavior depending on the wavelength of the initial imperfection, with short wavelengths promoting unstable behavior. Most notably, the example also illustrates the stabilizing effect of prestress, both as regards delaying the onset of instability to smaller imperfection wavelengths as well as accelerating the rate of decay in the stable case. 

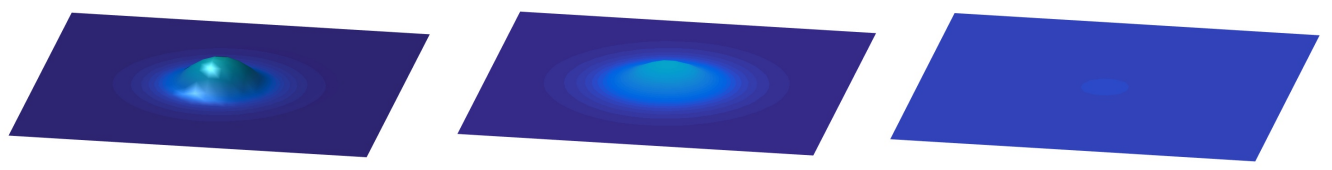

(a)
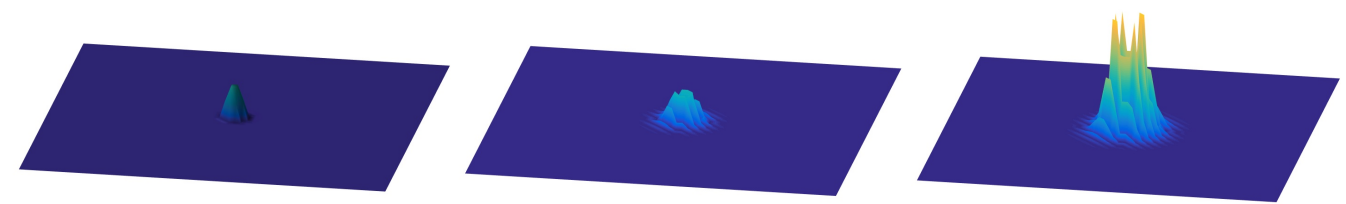

(b)
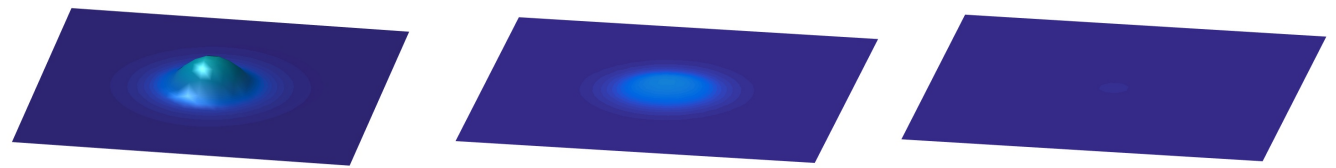

(c)

Figure 5: Computed time-evolution of a Li-LIPON interface with an initial imperfection. a) Stable growth of an initial perturbation with wavenumbers $k_{1}=k_{2}=0.5 \mu \mathrm{m}^{-1}$. Initial (left), intermediate (center), and final-planar state (right). The interface becomes ostensibly planar after $t=19,000 \mathrm{~s}$. b) Unstable growth of an initial perturbation with wavenumbers $k_{1}=k_{2}=2 \mu \mathrm{m}^{-1}$. Initial (left), intermediate (center), and clearly unstable state at $t=25 \mathrm{~s}$ (right). c) Stable growth of an initial perturbation with wavenumbers $k_{1}=k_{2}=0.5 \mu \mathrm{m}^{-1}$ and elastic prestress $\llbracket \sigma_{11}^{*} \rrbracket=100 \mathrm{MPa}$. Initial (left), intermediate (center) and final-planar state (right). The interface becomes ostensibly planar after $t=1,300$ s. 


\section{Stability analysis}

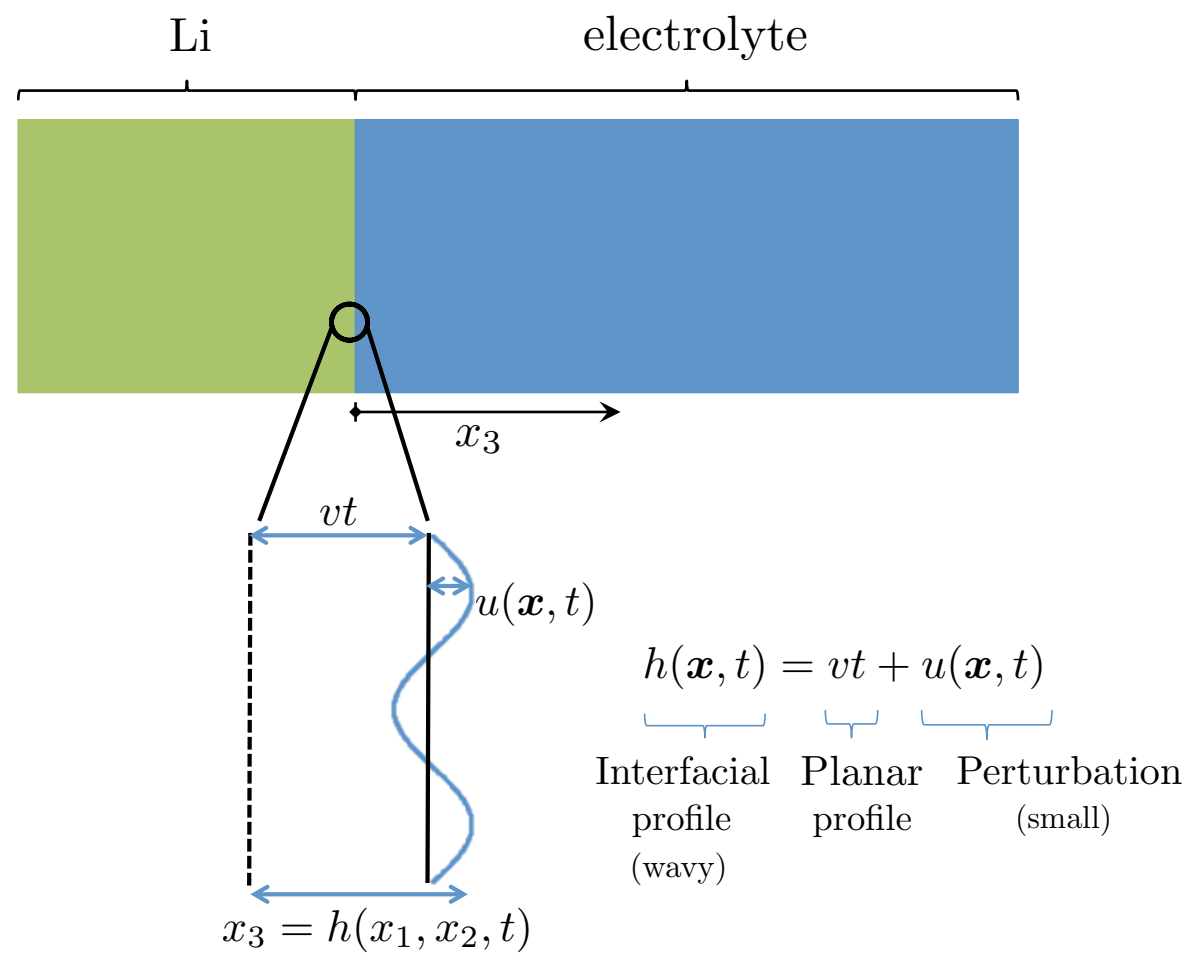

Figure 6: Small perturbation on the planar moving interface. Representation of the initial planar interface (black dashed line), the moving planar interface (black solid line) and the wavy interface (blue solid line).

The conditions under which the growth of a planar electrode/electrolyte interface is stable or unstable can be ascertained by means of a linear stability analysis. To this end, we consider the ansatz

$$
u(\boldsymbol{x}, t)=A \mathrm{e}^{-\lambda t} \mathrm{e}^{-i \boldsymbol{k} \cdot \boldsymbol{x}}
$$

where $\boldsymbol{k}$ is the wavenumber vector of the perturbation, and $\lambda>0$ and $\lambda<0$ correspond to decay and growth in time, respectively. Inserting this ansatz into the governing equation (37) we obtain the characteristic equation

$$
\lambda=\left(D_{1}+D_{3}\right)|\boldsymbol{k}|^{2}-D_{2}|\boldsymbol{k}|^{4}+D_{4} \hat{G}_{i k}(\boldsymbol{k}) \llbracket \sigma_{i \beta}^{*} \rrbracket \llbracket \sigma_{k \delta}^{*} \rrbracket k_{\beta} k_{\delta} .
$$


This relation gives the rate of growth of perturbations according to their wavenumbers. We observe that particle-exchange diffusion, interfacial energy and elastic terms are quadratic in $\boldsymbol{k}$, whereas the capillarity-driven interfacial term is quartic in $\boldsymbol{k}$. The quadratic terms dominate for small $\boldsymbol{k}$, or large wavelengths, whereas the quartic term dominates for large $\boldsymbol{k}$, or small wavenumbers.

In the absence of elastic prestraining, i. e, if $\sigma_{i j}^{*}=0$, it follows from (44) that growth is always stable for $D_{2}<0$, corresponding to capillaritydriven interfacial antidiffusion. For conventional capillarity-driven interfacial diffusion, $D_{2}>0$, planar interfaces are stable for small $|\boldsymbol{k}|$ but loose stability when

$$
\left(D_{1}+D_{3}\right)|\boldsymbol{k}|^{2}-D_{2}|\boldsymbol{k}|^{4}=0
$$

or

$$
|\boldsymbol{k}|=\sqrt{\frac{D_{1}+D_{3}}{D_{2}}} \equiv k_{c},
$$

where $k_{c}$ is the critical wavenumber for loss of stability in the unstressed case. For instance, for the Li-LIPON system characterized in Sec. 2.5, we calculate $k_{c} \approx 1.12 \mu \mathrm{m}^{-1}$, which corresponds to a critical wavelength of $L=5.6 \mu \mathrm{m}$.

We also note that the elastic stress always contributes a positive number to the characteristic equation (44) and, therefore, it has the effect of raising the value of the critical wavenumber $k_{c}$, see Fig. 7. Consequently, prestress always has a stabilizing effect on the electrode-electrolyte interface, i. e., it tends to keep it planar. The analysis thus suggests that planar electrode-electrolyte interfaces can be stabilized over an arbitrary range of wavenumbers by subjecting them to a sufficiently large prestress.

Fig. 8 illustrates the effect of prestress on stability for the lithium/LIPON system under equi-biaxial prestress, $\llbracket \sigma_{11}^{*} \rrbracket=\llbracket \sigma_{22}^{*} \rrbracket$. By the symmetry of the system, the growth rate $\lambda$ depends on $|\boldsymbol{k}| \equiv k$ only. The stabilizing effect of prestress is evident from the figure. The critical wavenumber increases from $k_{c}=1.12 \mu \mathrm{m}^{-1}$ in the absence of prestress to $k_{c}=1.66 \mu \mathrm{m}^{-1}$ for the maximum stress jump considered $\llbracket \sigma_{11}^{*} \rrbracket=\llbracket \sigma_{22}^{*} \rrbracket=500 \mathrm{MPa}$, see Fig. 8. However, because of the high stiffness of LIPON the levels of prestress required to effect the stabilization are quite large. 


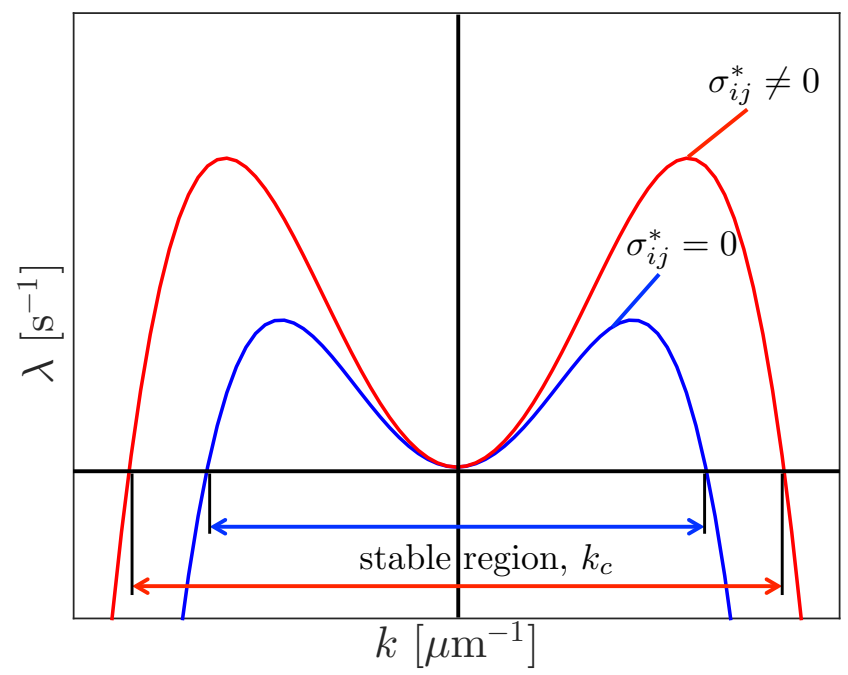

Figure 7: Effect of elastic prestress on the critical wavenumber $k_{c}$. Stable region in the absence (blue line) and in the presence (red line) of elastic stress. Stable growth $\lambda>0$ is observed for small wavenumbers, the region of which gets enlarged with applied stress. 


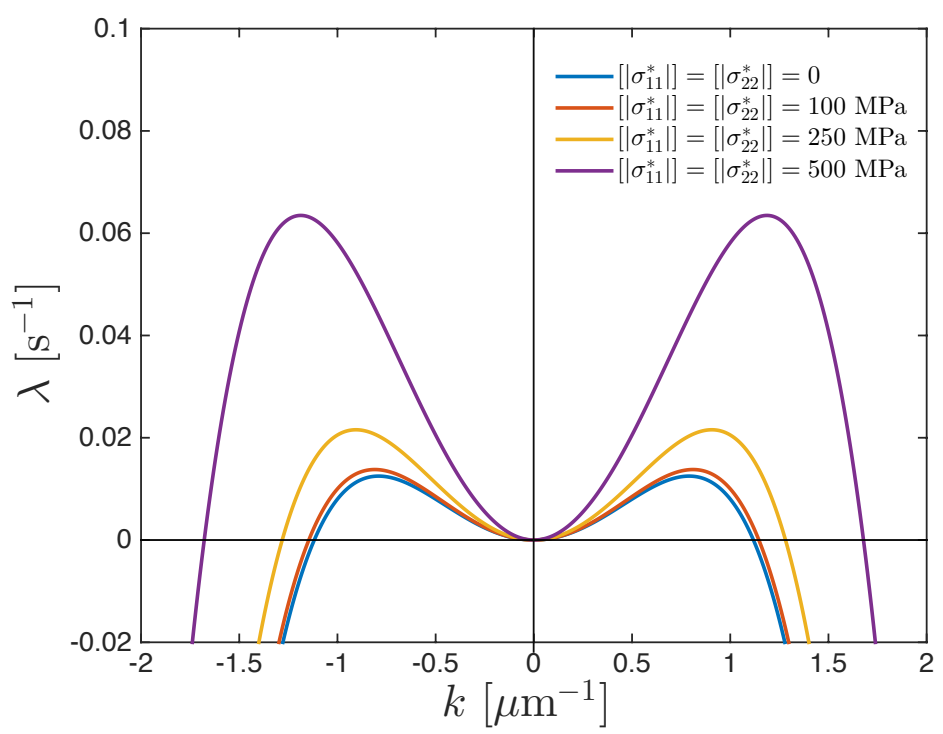

Figure 8: Effect of equi-biaxial prestress on the stability diagram of a Li-LIPON material system.

\section{Application to lithium/solid-state dioxolane-dimethoxy ethane electrolyte}

There is a severe paucity of experimental data at present for purposes of validation of the theory. A notable exception is the work of Mikhaylik et al. [40], who have reported on the effect of prestress for a system consisting of a lithium anode and a solid-state dioxolane-dimethoxy ethane (DOL+DME) electrolyte that does supply a modicum of validation. The specific electrolyte considered by Mikhaylik et al. [40] provides a high sulfide solubility needed for improved cathode operation and a stable solid electrolyte interface on the metallic lithium surface. If unstable, the configuration prevents the growth of long dendrites but a mossy or scallop like surface roughness still arises. This surface roughening, together with the reactivity of lithium, negatively impacts the safe operation of the battery.

In [40], different physical and chemical approaches to improve stability were investigated experimentally. In particular, thin layers of metallic lithium have been discharged to a depth of $100 \%$ and charged again. It was found, that the problem of roughening transition was practically eliminated through the application of pressure to the lithium anode surface. This effect is clearly 


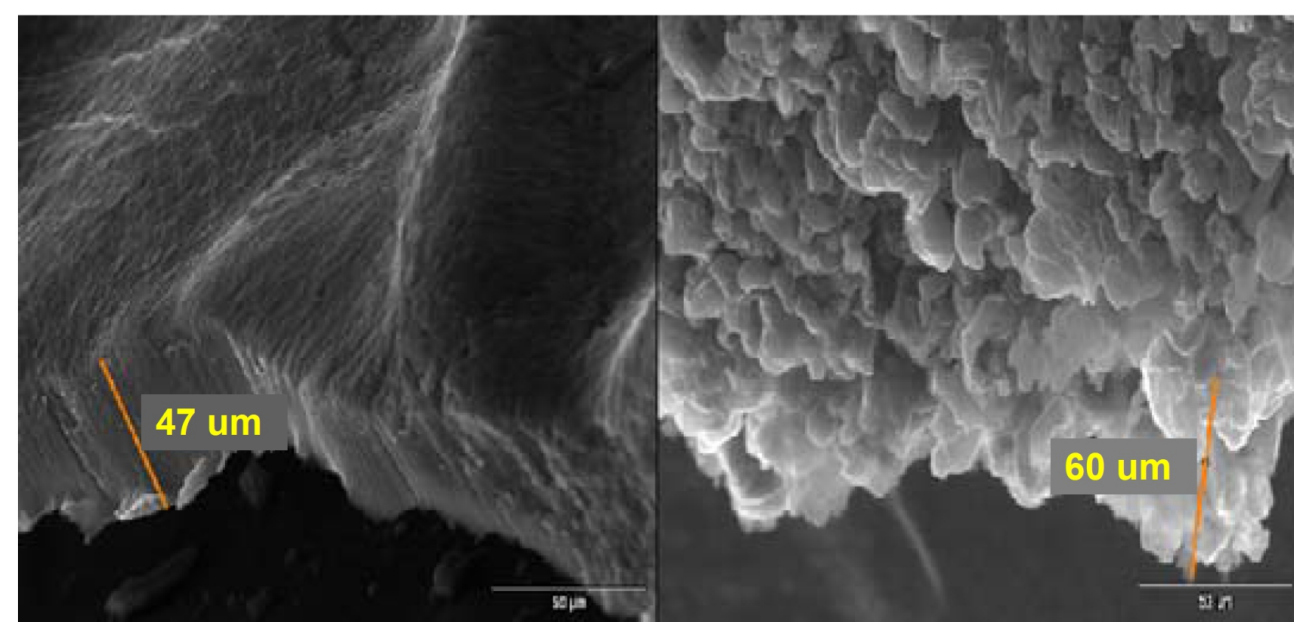

Figure 9: Lithium anodes after 50 cycles of charging and discharging: at uniaxial pressure of $1 \mathrm{MPa}$ (left) and without compression (right); photographs from [40].

evident in Fig. 9. The marked $47 \mu \mathrm{m}$ and $60 \mu \mathrm{m}$ distance bars indicate the thickness of the lithium layer after 50 cycles of depletion. The initial anode thickness was $50 \mu \mathrm{m}$ in both cases.

Unfortunately, the material constants required to apply the theory to the LI/DOL+DME electrolyte system are not all known. However, the observations of Mikhaylik et al. [40] set bounds on the values of those constants. Thus, Fig. 9b suggests that, in the absence of prestress, the interface roughens on lengthscales of the order of $25 \mu \mathrm{m}$ and less, which gives the estimate $k_{c}\left(\sigma_{33}=0\right) \approx 0.25 \mu \mathrm{m}^{-1}$. In view of (46), this estimate in turn gives $\left(D_{1}+D_{3}\right) / D_{2} \approx 0.01 \mu \mathrm{m}^{-2}$. By contrast, Fig. 9a, corresponding to a normal pressure of $\sigma_{33}=1 \mathrm{MPa}$, shows surface ripples on a scale of the order of $\mu \mathrm{m}$ or less, which gives the estimate $k_{c}\left(\sigma_{33}=1 \mathrm{MPa}\right) \approx 1.25 \mu \mathrm{m}^{-1}$. Assuming for DOL+DME a shear modulus of $1 \mathrm{MPa}$ and a bulk modulus of $500 \mathrm{MPa}$, as typical elastic constants of a soft polymer, a straightforward calculation gives a biaxial prestress jump of

$$
\llbracket \sigma_{11}^{*} \rrbracket=\llbracket \sigma_{22}^{*} \rrbracket=\left(\frac{c_{12}^{+}}{c_{11}^{+}}-\frac{c_{12}^{-}}{c_{11}^{-}}\right) \sigma_{33} \equiv \sigma^{*} \approx 0.15 \mathrm{MPa} .
$$

In order to derive a simple estimate of the elastic term in the characteristic equation (44), we note that, in view of the stiffness contrast between $\mathrm{Li}$ and DOL+DME we may approximate the interface Green's function by means 
the surface Green's function of Li, with the result

$$
\begin{aligned}
& \hat{G}_{11}(\boldsymbol{k})=\frac{1}{2 \mu|\boldsymbol{k}|^{3}}\left(2|\boldsymbol{k}|^{2}+k_{1}^{2}\left(\alpha^{-1}-2\right)\right), \\
& \hat{G}_{22}(\boldsymbol{k})=\frac{1}{2 \mu|\boldsymbol{k}|^{3}}\left(2|\boldsymbol{k}|^{2}+k_{2}^{2}\left(\alpha^{-1}-2\right)\right), \\
& \hat{G}_{12}(\boldsymbol{k})=\hat{G}_{21}(\boldsymbol{k})=\frac{k_{1} k_{2}}{2 \mu|\boldsymbol{k}|^{3}}\left(\alpha^{-1}-2\right),
\end{aligned}
$$

where

$$
\alpha=\frac{\lambda+\mu}{\lambda+2 \mu}=\frac{1}{2(1-\nu)},
$$

$\lambda$ and $\mu$ are Lamé constants, $\nu$ is Poisson's ratio and, for purposes of the present analysis, we idealize lithium as isotropic elastic. Using (47) and (48), we obtain

$$
D_{4} \hat{G}_{i k}(\boldsymbol{k}) \llbracket \sigma_{i \beta}^{*} \rrbracket \llbracket \sigma_{k \delta}^{*} \rrbracket k_{\beta} k_{\delta}=D_{4} \frac{\left(\sigma^{*}\right)^{2}}{2 \mu} \alpha^{-1}|\boldsymbol{k}|,
$$

and the characteristic equation (44) reduces to

$$
0=\left(D_{1}+D_{3}\right) k_{c}^{2}-D_{2} k_{c}^{4}+D_{4} \frac{\left(\sigma^{*}\right)^{2}}{2 \mu} \alpha^{-1} k_{c}
$$

Neglecting, as in the case of Li/LIPON, $D_{1}$ against $D_{3}$, using the preceding estimates for $\left(D_{1}+D_{3}\right) / D_{2}$ and $k_{c}\left(\sigma_{33}=1 \mathrm{MPa}\right)$, making the identification $\mu \sim c_{44}, \lambda=c_{12}$ and using the values of Table 1 for lithium, and recalling the definition (38) of $D_{4}$, we find the estimate $\gamma \approx 1 \times 10^{-6} \mathrm{~J} / \mathrm{m}^{2}$, which is within the normal range of interfacial energies for metal/polymer bonding.

We conclude that the application of a modest pressure in systems characterized by weak lithium/electrolyte interfaces results in a substantial reduction in the roughening of the lithium surface during cycling, effectively eliminating the instability problem, in agreement with the observations of Mikhaylik et al. [40].

\section{Summary and concluding remarks}

We have formulated a model of the growth of electrode-electrolyte interfaces in lithium batteries in the presence of an elastic prestress and used the model as a basis for assessing the effect of prestress on the stability of planar growth and the potential of prestress as a means of suppressing the formation 
of deleterious dendrites. The model accounts for the kinetics of $\mathrm{Li}^{+}$transport through the solid electrolyte (e. g., LIPON) and within the interface, the kinetics of $\mathrm{Li}^{+}$adsorption by the anode, electrostatics, and the elastic field. These fields are in principle coupled due to pressure-assisted diffusion, swelling of the intercalated electrolyte, and the Maxwell stress of the electrostatic field, and contribute jointly to the chemical potential driving the motion of interface. In particular, the elastic field is sensitive to the shape of the interface, with the result that its roughening may be self-sustaining and eventually lead to the runaway formation of dendrites. We have accounted for this feedback effect through an asymptotic analysis of the elastic field of a nearly flat interface between two semi-infinite elastic bodies similar to analyses of nearly flat surfaces and interfaces carried out by Srolovitz [63] and by Gao $[27,28]$. Direct numerical simulations immediately evince the possibility of stable and unstable growth depending on the choice of material constants and applied prestress. Linear stability analysis then results in explicit analytical expressions for the dependence of growth rates, and of the critical unstable wavelength for the interfacial roughening, on the state of stress and on fundamental parameters such as surface diffusivities, surface energy, deposition kinetics, and elastic moduli. Finally, we have examined the model in the light of the experimental observations of Mikhaylik et al. [40] concerned with the effect of pressure on a lithium/dioxolane-dimethoxy ethane electrolyte system and verified that, with reasonable choices of parameters and some calibration, the model accounts for the observation that a modest applied pressure results in a substantial reduction in the roughening of the lithium surface during cycling, effectively eliminating the instability.

The experimental observations of Mikhaylik et al. [40] and the present work, taken together, point to prestressing as a promising - and as yet untapped-tool for the design of lithium batteries. The model characterizes the relation between growth rates and stability ranges and key material properties, on which basis such properties can be selected or engineered so as to suppress the formation of dendrites. The model also quantifies the effect of prestress on planar-growth stability, which again opens the way for consideration of prestress in battery design and for identifying promising materials systems.

Evidently, a number of aspects of the model could benefit from further refinement. Thus, the model regards the lithium/electrolyte interface as sharp, in contrast to practical designs in which the interface may have considerable structure. Model extensions accounting from more practical designs are likely 
to extend the applicability of the model, albeit at the expense of increased complexity. In addition, some of the material constants that enter in the model are not known or known imperfectly. Here again, a more precise material characterization, be it on the basis of first-principles calculations or experimental measurement, should improve the predictiveness of the model. Finally, as already noted the elucidation of the effect of prestress on planargrowth stability of lithium/electrolyte interfaces is hampered by a severe lack of experimental data at present. Carefully designed experiments and adequate experimental data constitute conditio sine qua non for establishing the physical phenomenon itself and enabling model validation.

\section{Acknowledgements}

We gratefully acknowledge support from Robert Bosch GmbH through the Bosch Energy Research Network (BERN) Project No.: 07-15-CS13 and from the U.S. National Science Foundation through the Partnership for International Research and Education (PIRE) on Science at the Triple Point Between Mathematics, Mechanics and Materials Science, Award Number 0967140. We are indebted to Dr. Thomas Eckl, Dr. Felix Hildebrand and Dr. Ulrich Sauter, of Computational Materials Engineering, Renningen, Robert Bosch GmbH, 70465 Stuttgart, Germany, and to Dr. Boris Kozinsky, Research and Technology Center North America, Robert Bosch LLC, Cambridge, Massachusetts 02140, USA, for extended discussions and guidance.

\section{Appendix A. The elastic energy of a wavy interface}

The analysis of Section 2.3 shows that, to first order, the perturbation in the elastic field of two prestressed semi-infinite linear-elastic solids due to the introduction of a slight waviness in the otherwise planar interface is given by the solution of the linear-elastic problem of a planar interface subject to the distribution of tractions

$$
t_{i}=\llbracket \sigma_{i \beta}^{*} \rrbracket h, \beta
$$

where $\llbracket \sigma_{i \beta}^{*} \rrbracket$ is the jump in residual stress across the unperturbed interface and $h\left(x_{1}, x_{2}\right)$ is the height profile of the wavy interface. Since the initial residual stresses are in equilibrium, the excess energy due to the introduction of the waviness follows from the work-energy theorem as

$$
E^{\mathrm{ela}}=\int_{-\infty}^{\infty} \int_{-\infty}^{\infty} \frac{1}{2} \hat{t}_{i}\left(k_{1}, k_{2}\right) \hat{u}_{i}^{*}\left(k_{1}, k_{2}, 0\right) \mathrm{d} k_{1} \mathrm{~d} k_{2},
$$




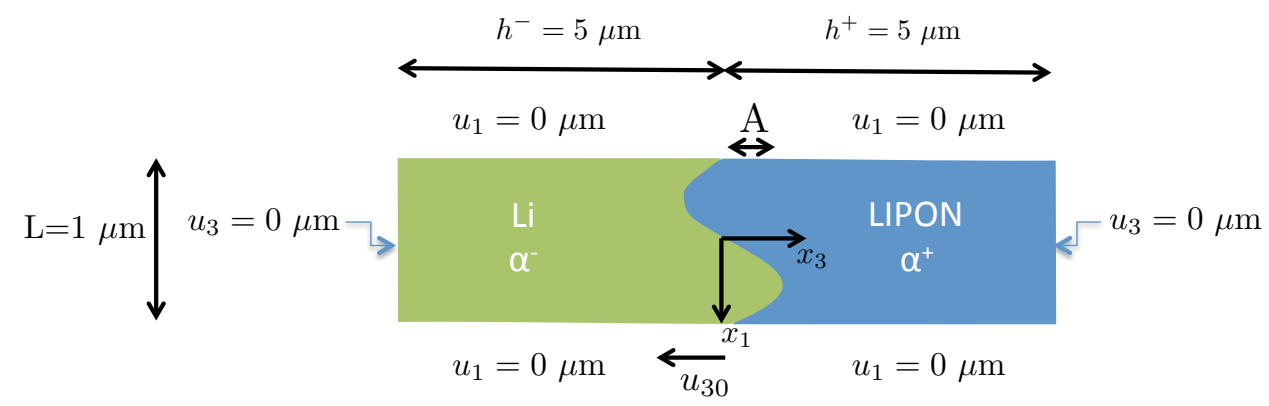

Figure A.10: Geometry of the single strip with different thermal expansion coefficients $\alpha^{ \pm}$ for each material region.

or

$$
E^{\text {ela }}=\int_{-\infty}^{\infty} \int_{-\infty}^{\infty} \frac{1}{2} \hat{G}_{i k}\left(k_{1}, k_{2}\right) \hat{t}_{i}\left(k_{1}, k_{2}\right) \hat{t}_{k}^{*}\left(k_{1}, k_{2}\right) \mathrm{d} k_{1} \mathrm{~d} k_{2} .
$$

Alternatively, an application of the inverse Fourier transform gives

$$
E^{\text {ela }}=\int_{\mathbb{R}^{2}} \int_{\mathbb{R}^{2}} \frac{1}{2} G_{i k}\left(\boldsymbol{x}-\boldsymbol{x}^{\prime}\right) t_{i}(\boldsymbol{x}) t_{k}\left(\boldsymbol{x}^{\prime}\right) \mathrm{d} \boldsymbol{x} \mathrm{d} \boldsymbol{x}^{\prime},
$$

which corresponds to the change in elastic energy with respect to the planar interface.

By way of verification of the linearized perturbation analysis, we consider the special case of an interface separating two linear-elastic solids with sinusoidal waviness in one direction, Fig. A.10. We then compare the excess energy predicted by (A.4) with the excess energy computed directly from a finite element analysis. For the waviness under consideration, the problem conveniently reduces to plane strain. Under these conditions, Clapeyron's theorem gives

$$
E=\int_{-L / 2}^{+L / 2} \frac{1}{2} t_{i}\left(x_{1}\right) u_{i}\left(x_{1}, 0\right) d x_{1},
$$

where $L$ is the wavelength of the sinusoidal interfacial profile. The displace- 
ment $u_{i}$ at the interface is in turn given by

$$
\begin{aligned}
u_{i}\left(x_{1}, 0\right) & =\int_{-\infty}^{+\infty} \int_{-\infty}^{+\infty} G_{i k}\left(x_{1}-x_{1}^{\prime}, x_{2}^{\prime}\right) t_{k}\left(x_{1}^{\prime}\right) d x_{1}^{\prime} d x_{2}^{\prime} \\
& =\int_{-\infty}^{+\infty} g_{i k}\left(x_{1}-x_{1}^{\prime}\right) t_{k}\left(x_{1}^{\prime}\right) d x_{1}^{\prime} \\
& =\left(g_{i k} * t_{k}\right)\left(x_{1}\right)
\end{aligned}
$$

where

$$
g_{i k}\left(x_{1} ; x_{1}^{\prime}\right)=\int_{-\infty}^{+\infty} G_{i k}\left(x_{1}-x_{1}^{\prime}, x_{2}^{\prime}\right) d x_{2}^{\prime}
$$

and $G_{i k}$ is the Green's function matrix. By periodicity, we can equivalently write the energy in Fourier series representation as

$$
E=L \sum_{m=-\infty}^{m=+\infty} \frac{1}{2} \hat{t}_{i}(m) \hat{u}_{i}^{*}(m)
$$

where

$$
\begin{aligned}
& \hat{t}_{i}(m)=\frac{1}{L} \int_{-L / 2}^{+L / 2} t_{i}\left(x_{1}\right) \mathrm{e}^{-i(2 \pi m / L) x_{1}} d x_{1}, \\
& \hat{u}_{i}(m)=\frac{1}{L} \int_{-L / 2}^{+L / 2} u_{i}\left(x_{1}\right) \mathrm{e}^{-i(2 \pi m / L) x_{1}} d x_{1}
\end{aligned}
$$

are Fourier series coefficients for the traction and the displacement, respectively, with $m \in \mathbb{Z}^{n}$. From the periodicity of the traction function we additionally have

$$
\left(g_{i k} * t_{k}\right)(m)=\hat{g}_{i k}\left(\frac{2 \pi m}{L}\right) \hat{t}_{k}(m) .
$$

By virtue of these identities, (A.8) reduces to

$$
E=L \sum_{m=-\infty}^{m=+\infty} \frac{1}{2} \hat{g}_{i k}\left(\frac{2 \pi m}{L}\right) \hat{t}_{i}(m) \hat{t}_{k}^{*}(m) .
$$

We additionally have that

$$
\hat{g}_{i k}\left(k_{1}\right)=\hat{G}_{i k}\left(k_{1}, 0\right),
$$

since the profile is wavy in the $x_{1}$-direction and constant in the $x_{2}$-direction. For a sinusoidal profile of the form

$$
h=A \sin \left(\frac{2 \pi}{L} x_{1}\right),
$$



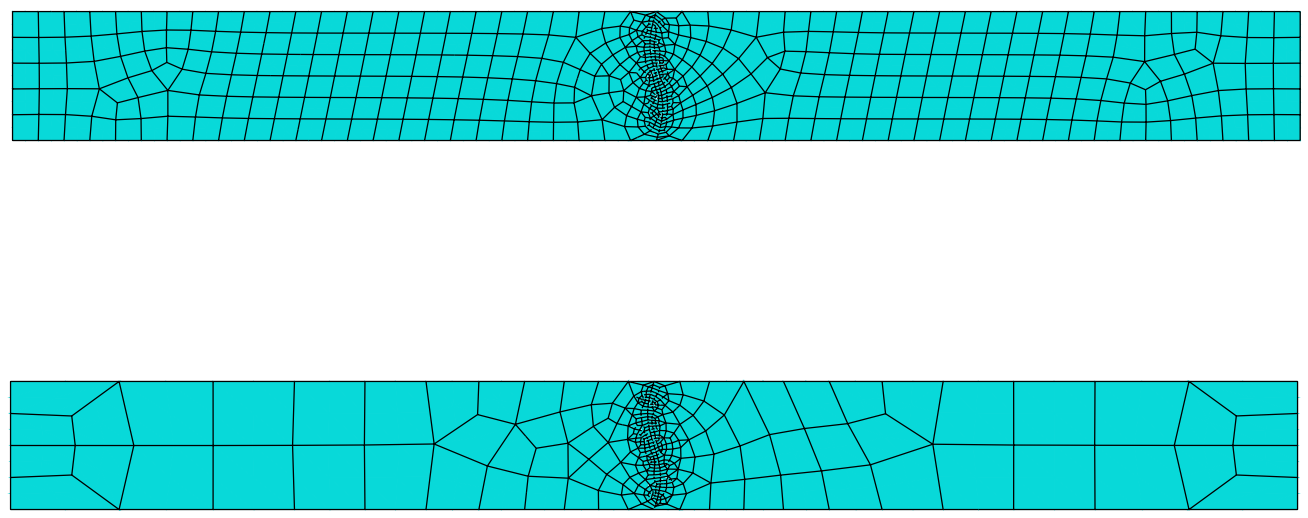

Figure A.11: Exemplary finite element mesh for the case of a wavy interface with amplitude $A=0.05[\mu \mathrm{m}]$. The original mesh (left) consists of 1,784 nodes and 563 elements and the optimized mesh (right) consists of 1011 nodes and 326 elements.

and assuming a jump in $\sigma_{11}^{*}$ only, eq. (A.11) finally reduces to, explicitly,

$$
E^{\mathrm{ela}}=\frac{A^{2} L}{8}\left(\frac{2 \pi}{L}\right)^{2} \hat{G}_{11}\left(\frac{2 \pi}{L}, 0\right) \llbracket \sigma_{11}^{*} \rrbracket^{2} .
$$

The quadratic dependence of the excess energy on the amplitude $A$ is noteworthy.

The explicit formula (A.14) for the excess energy can now be conveniently taken as a basis for verification of the linearized perturbation approach. Specifically, we compare the formula (A.14) with direct finite-element calculations over a range of amplitudes $A$. A sample finite-element model used in calculations is shown in Fig. A.11. By periodicity, we restrict the model to one wavelength and imposed periodic boundary conditions. In addition, the vertical displacement is constraint on the top and bottom segments of the boundary. In calculations, an equilibrated state of residual prestress is induced by recourse to thermal expansion. The corresponding residual prestresses are

$$
\sigma_{i j}^{ \pm}=c_{i j k l}^{ \pm}\left(\epsilon_{k l}^{ \pm}-\alpha^{ \pm} \Delta T \delta_{k l}\right)
$$

where $\Delta T$ is a prescribed temperature increment and $\alpha^{ \pm}$are thermal expan- 
sion coefficients. By compatibility and the boundary conditions, we have

$$
\epsilon_{33}^{+}=\frac{w_{0}}{H / 2}, \quad \epsilon_{33}^{-}=\frac{-w_{0}}{H / 2},
$$

where $H$ is the height of domain and $w_{0}$ is the transverse displacement of the planar interface. In addition, traction equilibrium at the interface gives

$$
\sigma_{33}^{+}=\sigma_{33}^{-} .
$$

The preceding equations determine the elastic field of the planar interface, including the elastic energy. In addition, a trite calculation gives

$$
\sigma_{11}^{ \pm}=c_{12}^{ \pm} \epsilon_{33}^{ \pm}-\left(c_{11}^{ \pm}+2 c_{12}^{ \pm}\right) \alpha^{ \pm} \Delta T
$$

for the discontinuous prestress component across the interface.

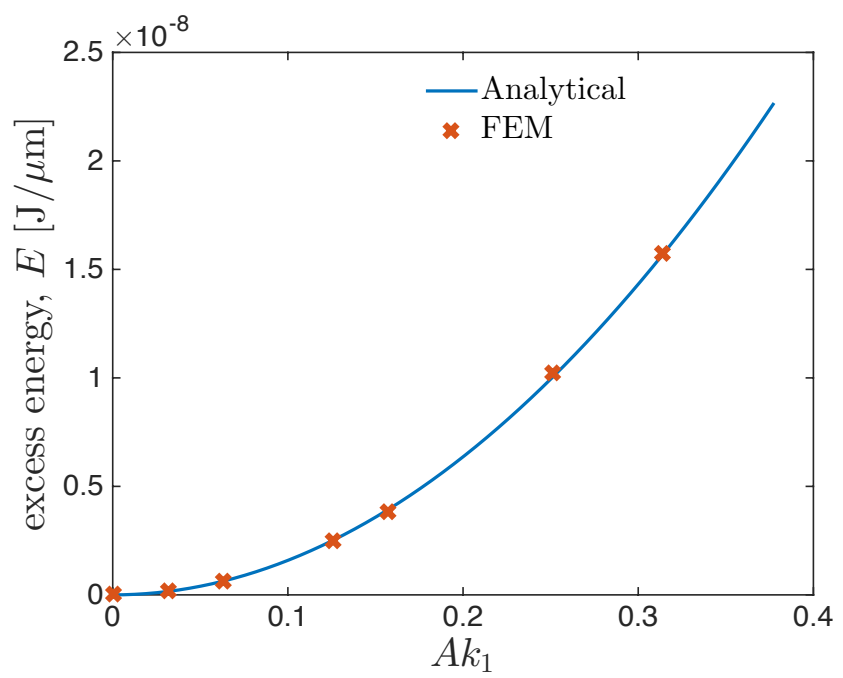

Figure A.12: Comparison plot of the excess of the elastic energy, calculated analytically and numerically (FEM), as a function of the normalized amplitude of the perturbation.

Sample calculations are carried out assuming elastic constants for LIPON $(+)$ and $\mathrm{Li}(-)$ as in Table 1. The thermal expansion coefficients assumed in the calculations are $\alpha^{+}=7 \cdot 10^{-5} / \mathrm{K}$ for LIPON and for lithium $\alpha^{-}=4.6$. 
$10^{-5} / \mathrm{K}$. We also assume a temperature increment $\Delta T=100 \mathrm{~K}$. The specific dimensions of the model used in calculations are also shown in Fig. A.10. The numerical excess energy at non-zero waviness amplitude $A$ is computed directly by subtracting from the elastic energy of the wavy interface the elastic energy of the planar interface, as provided by the preceding analysis. A range of mesh sizes is considered in order to insured results that are converged with respect to the mesh, cf. [46]. The calculations are carried out used the commercial code ABAQUS [14]. The corresponding analytical excess energy is computed by inserting (A.18) into (A.14).

Fig. A.12 shows a comparison of computed and analytical values of the excess elastic energy over a range of normalized amplitudes of the interface waviness. As may be seen from the figure, the agreement between theory and calculations is excellent, which effectively verifies the correctness of the linearized perturbation approach.

\section{Appendix B. Derivation of interfacial Green's function}

The solution of problems of linear elasticity by means of Green's function is classical, see e. g. [41, 45]. The Green's function for isotropic-elastic bimaterial solids was derived in $[19,25]$. Expressions for anisotropic materials can be found in $[4,44,65,66,68]$. For completeness, the requisite interfacial Green's function appearing in (32) is derived explicitly in this appendix using the Fourier transform. To this end, we consider a planar interface separating two semi-infinite linear-elastic solids and subject to prescribed tractions $\boldsymbol{t}$. The interfacial Green's function follows from the special case in which $\boldsymbol{t}$ is a point force applied to the interface.

With the interface at $x_{3}=0$ the governing equations are

$$
\begin{array}{lr}
\sigma_{i j, j}^{ \pm}=0, & \pm x_{3}>0, \\
\sigma_{i j}^{ \pm}=c_{i j k l}^{ \pm} \epsilon_{k l}^{ \pm}, & \pm x_{3}>0, \\
\epsilon_{i j}^{ \pm}=\frac{1}{2}\left(u_{i, j}^{ \pm}+u_{j, i}^{ \pm}\right), & \pm x_{3}>0, \\
u_{i}^{+}=u_{i}^{-}, & x_{3}=0, \\
\sigma_{i 3}^{+}-\sigma_{i 3}^{-}=t_{i}, & x_{3}=0, \\
u_{i}^{ \pm} \rightarrow 0, & x_{3} \rightarrow \pm \infty,
\end{array}
$$

where the labels $(+)$ and $(-)$ refer to the regions $\left\{x_{3}>0\right\}$ and $\left\{x_{3}<0\right\}$, respectively. We characterize the solution by means of the in-plane Fourier 
transform

$$
\hat{f}\left(k_{1}, k_{2}, x_{3}\right) \equiv \int_{-\infty}^{\infty} \int_{-\infty}^{\infty} f\left(x_{1}, x_{2}, x_{3}\right) \mathrm{e}^{-i\left(k_{1} x_{1}+k_{2} x_{2}\right)} d x_{1} d x_{2} .
$$

Applying this transform to the governing equations, we obtain

$$
\begin{array}{lr}
i \hat{\sigma}_{i \beta}^{ \pm} k_{\beta}+\hat{\sigma}_{i 3,3}^{ \pm}=0, & \pm x_{3}>0, \\
\hat{\sigma}_{i j}^{ \pm}=c_{i j k l}^{ \pm} \hat{\epsilon}_{k l}^{ \pm}, & \pm x_{3}>0, \\
\hat{\epsilon}_{\alpha \beta}^{ \pm}=\frac{1}{2}\left(i \hat{u}_{\alpha}^{ \pm} k_{\beta}+i \hat{u}_{\beta}^{ \pm} k_{\alpha}\right), & \pm x_{3}>0, \\
\hat{\epsilon}_{\alpha 3}^{ \pm}=\frac{1}{2}\left(\hat{u}_{\alpha, 3}^{ \pm}+i \hat{u}_{3}^{ \pm} k_{\alpha}\right), & \pm x_{3}>0, \\
\hat{\epsilon}_{33}^{ \pm}=\hat{u}_{3,3}^{ \pm}, & \pm x_{3}>0, \\
\hat{u}_{i}^{+}=\hat{u}_{i}^{-}, & x_{3}=0, \\
\hat{\sigma}_{i 3}^{+}-\hat{\sigma}_{i 3}^{-}=\hat{t}_{i}, & x_{3}=0, \\
\hat{u}_{i}^{ \pm} \rightarrow 0, & x_{3} \rightarrow \pm \infty,
\end{array}
$$

where, here and subsequently, Greek indices take the values $\{1,2\}$. Combining the above equations yields the second-order ODE problem

$$
\begin{aligned}
& A_{i k}^{ \pm}(\boldsymbol{k}) \hat{u}_{k, 33}^{ \pm}+i B_{i k}^{ \pm}(\boldsymbol{k}) \hat{u}_{k, 3}^{ \pm}+C_{i k}^{ \pm}(\boldsymbol{k}) \hat{u}_{k}^{ \pm}=0, \\
& \hat{u}_{i}^{+}(\boldsymbol{k}, 0)=\hat{u}_{i}^{-}(\boldsymbol{k}, 0), \\
& \hat{\sigma}_{i 3}^{+}(\boldsymbol{k}, 0)-\hat{\sigma}_{i 3}^{-}(\boldsymbol{k}, 0)=\hat{t}_{i}(\boldsymbol{k}), \\
& \hat{u}_{i}^{ \pm}\left(\boldsymbol{k}, x_{3}\right) \rightarrow 0, \quad x_{3} \rightarrow \pm \infty
\end{aligned}
$$

where

$$
\begin{aligned}
& A_{i k}^{ \pm}(\boldsymbol{k})=c_{i 3 k 3}^{ \pm}, \\
& B_{i k}^{ \pm}(\boldsymbol{k})=c_{i \beta k 3}^{ \pm} k_{\beta}+k_{\delta} c_{i 3 k \delta}^{ \pm}, \\
& C_{i k}^{ \pm}(\boldsymbol{k})=-c_{i \beta k \delta}^{ \pm} k_{\beta} k_{\delta} .
\end{aligned}
$$

Inserting trial solutions of the form

$$
\hat{u}_{i}^{ \pm}\left(\boldsymbol{k}, x_{3}\right)=U_{i}^{ \pm}(\boldsymbol{k}) \mathrm{e}^{\lambda(\boldsymbol{k}) x_{3}},
$$

we obtain the characteristic equation

$$
\operatorname{det}\left(\boldsymbol{A}^{ \pm} \lambda^{2}+i \boldsymbol{B}^{ \pm} \lambda+\boldsymbol{C}^{ \pm}\right)=0
$$


The (+) equation has three roots with negative real part $\left\{\lambda_{1}^{+}(\boldsymbol{k}), \lambda_{2}^{+}(\boldsymbol{k}), \lambda_{3}^{+}(\boldsymbol{k})\right\}$ and three corresponding unit eigenvectors $\left\{\boldsymbol{U}_{1}^{+}(\boldsymbol{k}), \boldsymbol{U}_{2}^{+}(\boldsymbol{k}), \boldsymbol{U}_{3}^{+}(\boldsymbol{k})\right\}$, whereas the (-) equation has three roots with positive real part $\left\{\lambda_{1}^{-}(\boldsymbol{k}), \lambda_{2}^{-}(\boldsymbol{k}), \lambda_{3}^{-}(\boldsymbol{k})\right\}$ and three corresponding unit eigenvectors $\left\{\boldsymbol{U}_{1}^{-}(\boldsymbol{k}), \boldsymbol{U}_{2}^{-}(\boldsymbol{k}), \boldsymbol{U}_{3}^{-}(\boldsymbol{k})\right\}$. By superposition,

$$
\hat{u}_{i}^{ \pm}\left(\boldsymbol{k}, x_{3}\right)=\sum_{p=1}^{3} V_{p}^{ \pm}(\boldsymbol{k}) U_{p i}^{ \pm}(\boldsymbol{k}) \mathrm{e}^{\lambda_{p}^{ \pm}(\boldsymbol{k}) x_{3}},
$$

where the six scalar amplitudes $\left\{V_{1}^{ \pm}(\boldsymbol{k}), V_{2}^{ \pm}(\boldsymbol{k}), V_{3}^{ \pm}(\boldsymbol{k})\right\}$ follow from the system defined by the six equations (B.4b) and (B.4c). In particular, by linearity we obtain the identity

$$
\hat{u}_{i}(\boldsymbol{k}, 0)=\hat{G}_{i k}(\boldsymbol{k}) \hat{t}_{k}(\boldsymbol{k}),
$$

where $\hat{G}_{i k}(\boldsymbol{k})$ is the Fourier transform of the surface Green's function.

By way of verification, we compare the Green's function with the expressions provided by Pan and Yuan [54, 53]. Specifically, we consider the case of a lithium-LIPON interface for purposes of comparison, cf. Table 1.
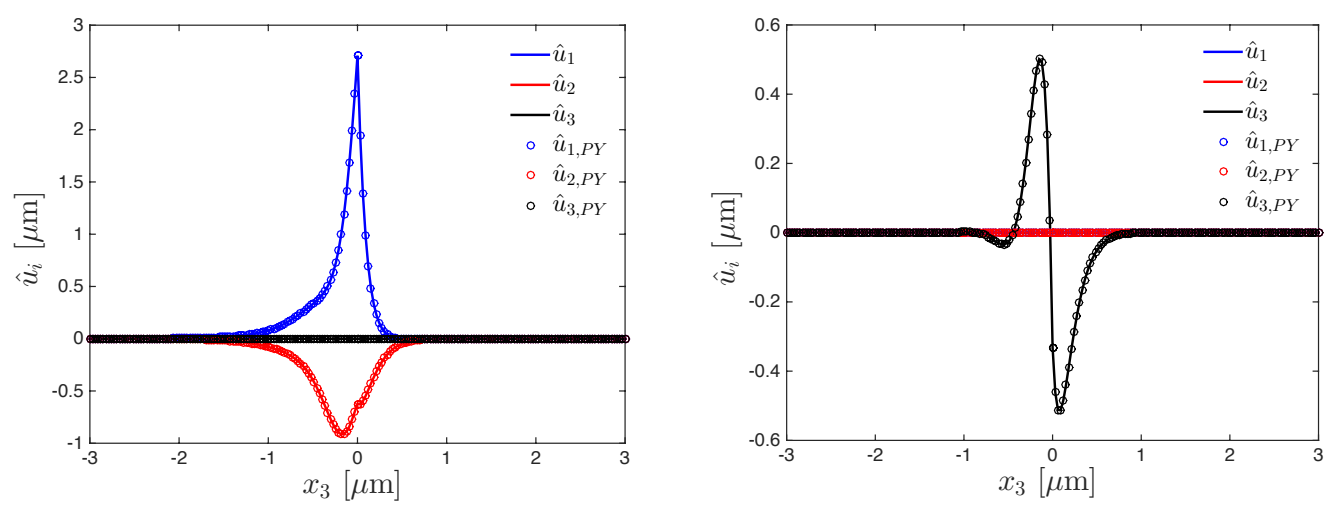

Figure B.13: Real (left) and imaginary (right) parts of the three components of the displacements, in the transformed domain, as a function of $x_{3}$ for applied traction $\hat{t}_{1}=[1,0,0]$. Comparison between the displacements of our model (solid lines) and the model of [54] (circular points).

Fig. B.13 shows the real and the imaginary part of the displacements as a function of the $x_{3}$ variable in the transformed domain, in the case of a unit load applied in the $x_{1}$-direction. In addition, Figure B.14 shows the corresponding out-of-plane components of stresses in the transformed domain. 

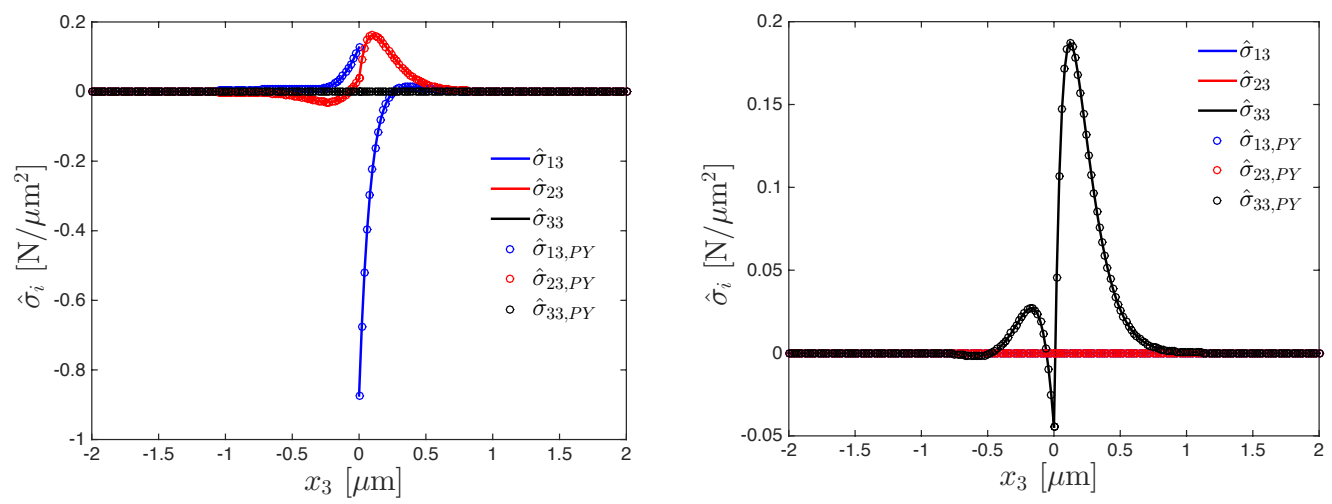

Figure B.14: Real (left) and imaginary (right) parts of the three out-of-plane components of the stresses, in the transformed domain, as a function of $x_{3}$ for applied traction $\hat{t}_{1}=$ $[1,0,0]$. Comparison between the stresses of our model (solid lines) and the model of [54] (circular points).

In both cases, there is perfect agreement between the present values and those reported by Pan and Yuan [54, 53]. As required, the displacements are continuous at the interface, $x_{3}=0$, and decay to zero away from the interface. By contrast, some components of the stress jump discontinuously at the interface due to the applied tractions.

\section{Bibliography}

[1] Rohan Akolkar. Mathematical model of the dendritic growth during lithium electrodeposition. Journal of Power Sources, 232:23-28, 2013.

[2] Michel Armand. Polymer solid electrolytes-an overview. Solid State Ionics, 9:745-754, 1983.

[3] Asghar Aryanfar, Daniel Brooks, Boris V Merinov, William A Goddard III, Agustin J Colussi, and Michael R Hoffmann. Dynamics of lithium dendrite growth and inhibition: Pulse charging experiments and monte carlo calculations. The Journal of Physical Chemistry Letters, 5(10):1721-1726, 2014.

[4] D. M. Barnett and J. Lothe. Line force loadings on anisotropic halfspaces and wedges. Physica Norvegica, 8(1):13-22, 1975. 
[5] J. L. Barton and Jo'M Bockris. The electrolytic growth of dendrites from ionic solutions. Proceedings of the Royal Society of London. Series A. Mathematical and Physical Sciences, 268(1335):485-505, 1962.

[6] M. Z. Bazant. Theory of chemical kinetics and charge transfer based on nonequilibrium thermodynamics. Accounts of Chemical Research, 46(5):1144-1160, 2013.

[7] Rangeet Bhattacharyya, Baris Key, Hailong Chen, Adam S Best, Anthony $\mathrm{F}$ Hollenkamp, and Clare $\mathrm{P}$ Grey. In situ nmr observation of the formation of metallic lithium microstructures in lithium batteries. Nature materials, 9(6):504-510, 2010.

[8] Esther Bohn, Thomas Eckl, Marc Kamlah, and Robert McMeeking. A model for lithium diffusion and stress generation in an intercalation storage particle with phase change. Journal of The Electrochemical Society, 160(10):A1638-A1652, 2013.

[9] Allan F Bower. Applied mechanics of solids. CRC press, 2009.

[10] S Chandrashekar, Nicole M Trease, Hee Jung Chang, Lin-Shu Du, Clare P Grey, and Alexej Jerschow. ${ }^{7}$ li mri of li batteries reveals location of microstructural lithium. Nature materials, 11(4):311-315, 2012.

[11] J. N. Chazalviel. Electrochemical aspects of the generation of ramified metallic electrodeposits. Physical review A, 42(12):7355, 1990.

[12] Jake Christensen, Paul Albertus, Roel S. Sanchez-Carrera, Timm Lohmann, Boris Kozinsky, Ralf Liedtke, Jasim Ahmed, and Aleksandar Kojic. A critical review of li/air batteries. Journal of the Electrochemical Society, 159(2):R1-R30, 2011.

[13] S. Das Sarma and S. V. Ghaisas. Solid-on-solid rules and models for nonequilibrium growth in $2+1$ dimensions. Physical Review Letters, 69(26):3762-3765, 1992.

[14] SIMULIA Dassault Systems. Abaqus FEA software - Finite Element Analysis / Standard user's Manual. Hibbitt, Karlsson \& Sorensen, 2001.

[15] AR Despic and Konstantin I Popov. Transport-controlled deposition and dissolution of metals, pages 199-313. Springer, 1972. 
[16] J. W. Diggle, A. R. Despic, and Jo'M Bockris. The mechanism of the dendritic electrocrystallization of zinc. Journal of The Electrochemical Society, 116(11):1503-1514, 1969.

[17] Mickael Dolle, Lucas Sannier, Bernard Beaudoin, Michel Trentin, and Jean-Marie Tarascon. Live scanning electron microscope observations of dendritic growth in lithium/polymer cells. Electrochemical and solidstate letters, 5(12):A286-A289, 2002.

[18] Marc Doyle, Thomas F. Fuller, and John Newman. Modeling of galvanostatic charge and discharge of the lithium/polymer/insertion cell. Journal of the Electrochemical Society, 140(6):1526-1533, 1993.

[19] John Dundurs and Miklas Hetenyi. Transmission of force between two semi-infinite solids. Journal of Applied Mechanics, 32(3):671-674, 1965.

[20] David R. Ely and R. Edwin Garcia. Heterogeneous nucleation and growth of lithium electrodeposits on negative electrodes. Journal of the Electrochemical Society, 160(4):A662-A668, 2013.

[21] Computational Materials Engineering. private communication. Robert Bosch GmbH, 70465 Stuttgart, Germany, 2015.

[22] I. Epelboin, M. Froment, M. Garreau, J. Thevenin, and D. Warin. Behavior of secondary lithium and aluminumlithium electrodes in propylene carbonate. Journal of The Electrochemical Society, 127(10):21002104, 1980.

[23] W.A. Goddard et al. Caltech, private communication.

[24] E Eweka, JR Owen, and A Ritchie. Electrolytes and additives for high efficiency lithium cycling. Journal of power sources, 65(1):247-251, 1997.

[25] N Fares and Victor C Li. General image method in a plane-layered elastostatic medium. ASME, Transactions, Journal of Applied Mechanics, 55:781-785, 1988.

[26] T. R. Ferguson and M. Z. Bazant. Nonequilibrium thermodynamics of porous electrodes. Journal of the Electrochemical Society, 159(12):A1967-A1985, 2012. 
[27] Huajian Gao. Stress concentration at slightly undulating surfaces. Journal of the Mechanics and Physics of Solids, 39(4):443-458, 1991.

[28] Huajian Gao. Some general properties of stress-driven surface evolution in a heteroepitaxial thin film structure. Journal of the Mechanics and Physics of Solids, 42(5):741-772, 1994.

[29] S. V. Ghaisas and S. Dassarma. Surface-diffusion length under kinetic growth-conditions. Physical Review B, 46(11):7308-7311, 1992.

[30] Katherine J Harry, Daniel T Hallinan, Dilworth Y Parkinson, Alastair A MacDowell, and Nitash P Balsara. Detection of subsurface structures underneath dendrites formed on cycled lithium metal electrodes. Nature materials, 13(1):69-73, 2014.

[31] E. G. Herbert, Wyatt E. Tenhaeff, Nancy J. Dudney, and G. M. Pharr. Mechanical characterization of lipon films using nanoindentation. Thin Solid Films, 520(1):413-418, 2011.

[32] C. Herring. Surface tension as a motivation for sintering. In w. E. Kingston, editor, The Physics of Powder Metallurgy. McGrawHill, New York, 1951.

[33] Toshiro Hirai, Isamu Yoshimatsu, and Junichi Yamaki. Influence of electrolyte on lithium cycling efficiency with pressurized electrode stack. Journal of The Electrochemical Society, 141(3):611-614, 1994.

[34] Kiyoshi Kanamura, Soshi Shiraishi, and Zenichiro Takehara. Electrochemical deposition of very smooth lithium using nonaqueous electrolytes containing hf. Journal of The Electrochemical Society, 143(7):2187-2197, 1996.

[35] Z. W. Lai and S. Das Sarma. Kinetic growth with surface relaxation - continuum versus atomistic models. Physical Review Letters, 66(18):2348-2351, 1991.

[36] Z.-W. Lai and S. Das Sarma. Kinetic growth with surface relaxation: Continuum versus atomistic models. Phys. Rev. Lett., 66:2348-2351, May 1991. 
[37] A. Latz and J. Zausch. Thermodynamic consistent transport theory of li-ion batteries. Journal of Power Sources, 196(6):3296-3302, 2011.

[38] Lucie Le Van-Jodin, Frederique Ducroquet, Frederic Sabary, and Isabelle Chevalier. Dielectric properties, conductivity and $l i+$ ion motion in lipon thin films. Solid State Ionics, 253:151-156, 2013.

[39] Alan C. Luntz, Johannes Voss, and Karsten Reuter. Interfacial challenges in solid-state li ion batteries. J. Phys. Chem. Lett., 6(22):45994604, 2015.

[40] Yuriy V. Mikhaylik, Igor Kovalev, Riley Schock, Karthikeyan Kumaresan, Jason Xu, and John Affinito. High energy rechargeable li-s cells for ev application: status, remaining problems and solutions. ECS Transactions, 25(35):23-34, 2010.

[41] Raymond D. Mindlin. Force at a point in the interior of a semi-infinite solid. Journal of Applied Physics, 7(5):195-202, 1936.

[42] Charles Monroe and John Newman. Dendrite growth in lithium/polymer systems: A propagation model for liquid electrolytes under galvanostatic conditions. Journal of The Electrochemical Society, 150(10):A1377-A1384, 2003.

[43] W. W. Mullins. Theory of thermal grooving. Japanese Journal of Applied Physics, 28:333, 1957.

[44] Toshio Mura. Micromechanics of defects in solids, volume 3. Springer Science and Business Media, 1987.

[45] N. I. Mußchelischwili. Einige Grundaufgaben zur mathematischen Elastizitätstheorie. Fachbuchverlag Leipzig, 1971.

[46] Panagiotis Philippos Natsiavas. Stability of Electrode-Electrolyte Interfaces during Charging in Lithium Batteries. PhD thesis, California Institute of Technology, 2016.

[47] E. B. Nauman and D. Q. He. Nonlinear diffusion and phase separation. Chemical Engineering Science, 56:19992018, 2001.

[48] John Newman and Karen E Thomas-Alyea. Electrochemical systems. John Wiley and Sons, 2012. 
[49] Tetsuo Nishida, Kei Nishikawa, M Rosso, and Yasuhiro Fukunaka. Optical observation of li dendrite growth in ionic liquid. Electrochimica Acta, 100:333-341, 2013.

[50] Warren C Oliver and Georges M Pharr. Measurement of hardness and elastic modulus by instrumented indentation: Advances in understanding and refinements to methodology. Journal of materials research, 19(01):3-20, 2004.

[51] Warren Carl Oliver and George Mathews Pharr. An improved technique for determining hardness and elastic modulus using load and displacement sensing indentation experiments. Journal of materials research, 7(06):1564-1583, 1992.

[52] M. Ortiz, E. A. Repetto, and H. Si. A continuum model of kinetic roughening and coarsening in thin films. Journal of the Mechanics and Physics of Solids, 47(4):697-730, 1999.

[53] E. Pan and B. Yang. Three-dimensional interfacial green's functions in anisotropic bimaterials. Applied Mathematical Modelling, 27(4):307-326, 2003.

[54] E. Pan and F. G. Yuan. Three-dimensional green's functions in anisotropic bimaterials. International Journal of Solids and Structures, 37(38):5329-5351, 2000.

[55] Emanuel Peled. The electrochemical behavior of alkali and alkaline earth metals in nonaqueous battery systems-the solid electrolyte interphase model. Journal of The Electrochemical Society, 126(12):20472051, 1979 .

[56] Rajlakshmi T. Purkayastha and Robert M. McMeeking. An integrated 2-d model of a lithium ion battery: the effect of material parameters and morphology on storage particle stress. Computational Mechanics, 50(2):209-227, 2012.

[57] C. Ratsch, M. D. Nelson, and A. Zangwill. Theory of strained-layer epitaxial-growth near step flow. Physical Review B, 50(19):14489-14497, 1994. 
[58] C. Ratsch, A. Zangwill, and P. Smilauer. Scaling of heteroepitaxial island sizes. Surface Science, 314(3):L937-L942, 1994.

[59] C. Ratsch, A. Zangwill, P. Smilauer, and D. D. Vvedensky. Saturation and scaling of epitaxial island densities. Physical Review Letters, 72(20):3194-3197, 1994.

[60] Thomas J Richardson and Guoying Chen. Solid solution lithium alloy cermet anodes. Journal of Power Sources, 174(2):810-812, 2007.

[61] K Saito, Y Nemoto, S Tobishima, and J Yamaki. Improvement in lithium cycling efficiency by using additives in lithium metal. Journal of power sources, 68(2):476-479, 1997.

[62] J. R. Smith and A. Zangwill. Ordering and roughening during the epitaxial growth of alloys. Physical Review Letters, 76(12):2097-2100, 1996.

[63] David J. Srolovitz. On the stability of surfaces of stressed solids. Acta Metallurgica, 37(2):621-625, 1989.

[64] Lars-Guran Sundstrom and Fritz H. Bark. On morphological instability during electrodeposition with a stagnant binary electrolyte. Electrochimica acta, 40(5):599-614, 1995.

[65] T. C. T. Ting. Anisotropic elasticity: Theory and applications. Oxford science publications. Oxford University Press, New York, 1996.

[66] T. C. T. Ting and Ven-Gen Lee. The three-dimensional elastostatic green's function for general anisotropic linear elastic solids. The Quarterly Journal of Mechanics and Applied Mathematics, 50(3):407-426, 1997.

[67] Shin-ichi Tobishima and Jun-ichi Yamaki. A consideration of lithium cell safety. Journal of Power Sources, 81:882-886, 1999.

[68] Fulvio Tonon, Ernian Pan, and Bernard Amadei. Green's functions and boundary element method formulation for $3 \mathrm{~d}$ anisotropic media. Computers and Structures, 79(5):469-482, 2001.

[69] Ulrich von Sacken, Eric Nodwell, Avtar Sundher, and JR Dahn. Comparative thermal stability of carbon intercalation anodes and lithium 
metal anodes for rechargeable lithium batteries. Journal of power sources, 54(2):240-245, 1995.

[70] W. C. West, J. F. Whitacre, and J. R. Lim. Chemical stability enhancement of lithium conducting solid electrolyte plates using sputtered lipon thin films. Journal of Power Sources, 126(1-2):134-138, 2004.

[71] Martin Winter, Wolfgang K Appel, Bernd Evers, Tomas Hodal, KaiChristian Müller, Ingo Schneider, Mario Wachtler, Markus R Wagner, Gerhard H Wrodnigg, and Jrgen O Besenhard. Studies on the anode/electrolyte interface in lithium ion batteries. Monatshefte für Chemie/Chemical Monthly, 132(4):473-486, 2001.

[72] Kang Xu. Nonaqueous liquid electrolytes for lithium-based rechargeable batteries. Chemical reviews, 104(10):4303-4418, 2004.

[73] Jun-ichi Yamaki, Shin-ichi Tobishima, Katsuya Hayashi, Keiichi Saito, Yasue Nemoto, and Masayasu Arakawa. A consideration of the morphology of electrochemically deposited lithium in an organic electrolyte. Journal of Power Sources, 74(2):219-227, 1998.

[74] Isamu Yoshimatsu, Toshiro Hirai, and Junichi Yamaki. Lithium electrode morphology during cycling in lithium cells. Journal of the Electrochemical Society, 135(10):2422-2427, 1988.

[75] X. H. Yu, J. B. Bates, G. E. Jellison, and F. X. Hart. A stable thinfilm lithium electrolyte: Lithium phosphorus oxynitride. Journal of the Electrochemical Society, 144(2):524-532, 1997.

[76] A. Zangwill. Some causes and a consequence of epitaxial roughening. Journal of Crystal Growth, 163:8-21, 1996.

[77] Guangyuan Zheng, Seok Woo Lee, Zheng Liang, Hyun-Wook Lee, Kai Yan, Hongbin Yao, Haotian Wang, Weiyang Li, Steven Chu, and Yi Cui. Interconnected hollow carbon nanospheres for stable lithium metal anodes. Nat Nano, 9(8):618-623, 2014. 